\title{
Collinearity issues in autoregressive models with time-varying serially dependent covariates
}

\author{
Sigert Ariens ${ }^{*}, 1,2$, Janne K. Adolf ${ }^{*}$, , and Eva Ceulemans* \\ *KU Leuven, Quantitative Psychology and Individual Differences, Faculty of Psychology and \\ Educational Sciences, Leuven, 3000, Belgium \\ ${ }^{1}$ sigert.ariens@kuleuven.be \\ 2Joint first author
}

\begin{abstract}
First-order autoregressive models are popular to assess the temporal dynamics of a univariate process. Researchers often extend these models to include time-varying covariates, such as contextual factors, to investigate how they moderate processes' dynamics. We demonstrate that doing so has implications for how well one can estimate the autoregressive and covariate effects, as serial dependence in the variables can imply predictor collinearity. This is a noteworthy contribution, since in current practice serial dependence in a time-varying covariate is rarely considered important. We first recapitulate the role of predictor collinearity for estimation precision in an ordinary least squares context, by discussing how it affects estimator variances, covariances and correlations. We then derive a general formula detailing how predictor collinearity in first-order autoregressive models is impacted by serial dependence in the covariate. We provide a simulation study to illustrate the implications of the formula for different types of covariates. The simulation results highlight when the collinearity issue becomes severe enough to hamper interpretation of the effects. We also show that the effect estimates can be biased in small samples (i.e., 50 time points). Implications for study design, the use of time as a predictor, and related model variants are discussed.
\end{abstract}

Keywords: Psychological dynamics, Autoregressive models, Collinearity, Time series analysis

\begin{tabular}{c|c}
\hline Version: & 2.0 \\
Date: & 07 July 2022 \\
Note: & This article has been accepted for publication in Multivariate Behavioral Research, \\
& published by Taylor and Francis. \\
\hline
\end{tabular}

\section{ACKNOWLEDGEMENTS}

This work was supported by a research fellowship from the German Research Foundation (DFG) awarded to Janne Adolf, by research grants from the Fund for Scientific Research-Flanders (FWO, Project No. G.074319N), the Research Council of KU Leuven (C14/19/054 and iBOF/21/090), awarded to Eva Ceulemans.

\section{DECLARATIONS OF INTEREST}

The authors declare the absence of any financial, intellectual, or other conflicts of interest which may have biased any aspect of this manuscript. 


\section{INTRODUCTION}

Autoregressive (AR) models constitute one of the most widely used methods for modeling serial dependence in intensive longitudinal data (ILD). Such models regress the present state of a univariate process on states of the same process at preceding time points by including lagged versions of the measured variable as predictor variables (e.g. Lütkepohl, 2005). In recent years, these models have seen extensive use in many fields of psychological research that focus on within-person dynamics. Examples range from developmental psychology (e.g. Degoy and Olmos, 2020), to psychopathology research (Groen et al., 2019) or experimental fields such as the analysis of eye-tracking data (Cho et al., 2018). Models for psychological research that extend the key ideas of AR models are published frequently (e.g. Gistelinck and Loeys, 2020, Asparouhov et al., 2018, Beltz and Gates, 2017, Krone et al., 2018, Bulteel et al., 2018).

To further introduce the key ideas of AR models, we turn to the field of affect dynamics in which AR models are routinely applied to ILD of a person's affective states (e.g., E. Hamaker et al., 2015. Ariens et al., 2020), to investigate how emotional processes behave over time at the within-person level (e.g., Kuppens, 2015). The field of affect dynamics typically uses first-order AR (i.e. AR(1)) models, implying that a single AR effect between successive process states (i.e., each process state is only regressed on the previous one) is sufficient to capture the serial dependence. The basic AR(1) model has three parameters which are all of theoretical interest for the study of affect dynamics. The intercept plays the same role as an intercept in classical regression models. Statistically it expresses the expected process state when all predictors equal 0. In AR(1) models it is often included to allow for non-zero means of affective processes, establishing the parameter's theoretical contribution (Bringmann et al., 2017.1. The residual variance, also called the innovation variance, is an important component of the variability of the emotional process. The emotional variability also depends on the party piece of the $\mathrm{AR}(1)$ model, which is that it allows process states at time $t$ to depend on the previous process state at time $t-1$, by means of the regression weight, or AR effect, of the lagged predictor. This AR effect is theoretically interpreted as an index of the extent to which an emotional process displays emotional inertia (Houben et al., 2015, Kuppens et al., 2010).

From the above, it is clear that psychologists tend to assign a close correspondence between the statistical parameters of $\mathrm{AR}(1)$ models and theoretical constructs. The powerful combination of these

\footnotetext{
${ }^{1}$ Note however that the intercept is not the same as the mean, unless one mean-centers the ILD before the analysis
} 
perceived relationships and the simplicity of the basic AR(1) model have spurred its widespread use. Whether there are inter-individual differences in the AR effect and whether these are meaningfully related to relevant person-level outcomes, has been a topic of considerable interest in recent publications (Koval et al., 2013, Bosley et al., 2019). However, its application comes with a number of assumptions which can be rather restrictive when applied in practice, such as the assumption that the process on which an AR(1) model is fitted displays a form of stability over time known as stationarity. This means that the process should display constant mean, variance, and autocorrelations over time. However, many substantive questions, (implicitly) assume that there is some form of change in affective dynamics over time (Bringmann et al., 2018, Adolf et al., 2017). The interest in the onset and development of pathological emotional processing (e.g. Lamers et al., 2018), an increase in average self-reported stress over time (e.g. Wang et al., 2020), a sudden change in emotional variability or stability following a major life event (e.g.Betz et al., 2020, Simons et al., 2020), or differences in the extent to which an emotional process displays inertia for instance (e.g. Waugh et al., 2017, Koval and Kuppens, 2012), can not be accommodated by the basic AR(1) model (see also Bringmann et al., 2017).

A popular route to model such changes in dynamics is the inclusion of relevant contextual information which may explain them, an approach which will be the topic of this paper. This can be achieved by appending observed time-varying covariates (e.g. different experimental stimuli or the occurrence of positive or negative events) to the basic AR(1) model (e.g. Lamers et al., 2018, Basinska and Gruszczynska, 2020 Kuppens, 2015, Brans et al., 2013). These covariates may excert their influence as main effects, altering the intercept and consequently the mean of the process at each time point. Next to including information on events or stimuli, adding time (or polynomials of time) as a separate predictor variable is often done to control for changes in mean over time (e.g. Jebb et al., 2015). Besides allowing for such intercept changes many researchers are interested in assessing changes in the AR effect (e.g. Koval and Kuppens, 2012). To this end, they include product terms of the lagged predictor and the covariate. Some researchers have also considered modeling changes in innovation variance (Adolf et al., 2017). It is important to realize that contextual predictors can be included in a lagged or contemporaneous way. In the lagged case, only the previous value of the covariate affects the process at time $t$, reflecting the idea that effects of the covariate unfold over time (Adolf et al., 2017). Contemporaneous effects imply that the influence of the covariate on the process is immediate. This might be more appropriate if the time intervals between measurements are large, while the effects of 
the covariate are rather short-lived (Schuurman et al.,2015), or almost immediate after their occurrence, for instance.

Although very useful from a substantive point of view, little attention has been paid to how including such time-varying covariates impacts the accuracy with which the parameters of the AR(1) model can be estimated. While omitting covariates can of course lead to biased estimates (e.g., of the AR effect), it is well known from the standard regression literature (e.g. Cohen et al., 2013) that estimation precision may be jeopardized in case of substantial collinearity between the predictors in the estimated model. Here, we use estimation precision to refer to estimator variances, and the related standard errors and confidence intervals, but also to estimator covariances and correlations, resulting in 'trade-off' behavior between estimators (cf. Leamer, 1973).

In this paper, we will show analytically and by means of simulations that collinearity is more of an issue in AR(1) models with time-varying covariates, as it can be amplified by the time series-nature of the variables in the model and their serial dependence. Specifically, using ordinary least squares (OLS) estimation theory, we first recapitulate in section 2.3 how the variances, covariances and correlations of regression coefficient estimators depend on the covariance and correlation (and hence collinearity) between predictors.

In section 3, we then analytically derive the predictor covariance as implied by the here-considered model (i.e., the covariance between the covariate and the lagged outcome), and show that it is affected by serial dependence in the covariate. This result holds regardless of the nature of the covariate. For the special case in which the covariate itself follows an $\mathrm{AR}(1)$ process, we can further show how serial dependence in the covariate, specifically its AR effect, affects the predictor covariance and correlation in interplay with the other model parameters. This establishes a direct link between (standardized) serial dependence in the covariate and predictor collinearity.

In section 4, we complement these results with a comprehensive simulation study, in which we verify and illustrate our analytical results examining estimation accuracy of AR(1) models with covariates of different formats and shapes and for plausible parameter effect sizes. We also show that in small samples, estimation precision can drop substantially, especially with highly autocorrelated predictors such as time trends. Furthermore, small-sample biases for the autoregressive effects can affect the other estimators in the case of high estimator correlations. In severe cases, the problem can be extensive enough to distort theoretical interpretation of the coefficients. In the discussion in Section 5, we reflect 
on the implications of these findings for study design and for extensions of the model that we studied.

\section{AUTOREGRESSIVE MODELS AND THEIR ESTIMATION}

\subsection{The basic $A R(1)$ model}

The basic AR(1) model assumes that the values of the process at each time point, $y_{t}$, with $t=$ $1, \ldots, T$ indexing the $T$ measurement occasions, can be predicted based on the values of the preceding observation, $y_{t-1}$. This model implies that only the most recent past is informative about the state of the process at time $t$. In equations, an AR(1) model is given by

$$
y_{t}=\beta_{0}+\beta_{1} y_{t-1}+\varepsilon_{t}, \text { with } \varepsilon_{t} \sim N\left(0, \sigma_{\varepsilon}^{2}\right),
$$

where $\beta_{0}$ is the intercept, $\beta_{1}$ is the AR effect, and $\varepsilon_{t}$ is the error term capturing the so-called process innovations over time. As their name suggests, the innovations are assumed to be independent of past process states up to and including $y_{t-1}$, serially independent, and distributed according to a zero-mean normal distribution with constant variance ${ }^{2}, \sigma_{\varepsilon}^{2}$. In addition, the AR effect must be smaller than 1 in absolute value for an $\mathrm{AR}(1)$ model to display covariance stationarity. In that case, the process

displays a constant mean, which is given by $\mu_{y}=\frac{\beta_{0}}{\left(1-\beta_{1}\right)}$, constant variance, given by $\sigma_{y}^{2}=\frac{\sigma_{\varepsilon}^{2}}{\left(1-\beta_{1}\right)^{2}}$, and a constant autocorrelation $\beta_{1}$ (Hamilton, 1994). The innovations can then be thought of as perturbations, which drive the process away from its mean and feed forward through time due to the presence of the AR effect. Specifically, the size of the AR effect indicates how far perturbations feed forward and how quickly the process returns to its mean. The closer the AR coefficient is to 1, the longer it will take for the effect of an innovation to "die out", and the slower the process will revert to its mean (Hamilton, 1994, p. 54).

\subsection{The AR(1) model with a single time-varying covariate}

As mentioned in the introduction, time-varying covariates are often included in an AR(1) model in order to assess whether and how the model parameters depend on the contextual information reflected in the covariate scores. These effects can be conceived as contemporaneous or as lagged. We limit the following presentation to the case of a single covariate for reasons of simplicity. Specifically, to

\footnotetext{
${ }^{2}$ implying homoskedasticity (Wooldridge, 2012, p. 243)
} 
investigate how the intercept changes as a function of the covariate scores at the preceding observation (i.e., a lagged effect), one can include the main effect of the lagged time-varying covariate:

$$
y_{t}=\beta_{0}+\beta_{1} y_{t-1}+\beta_{2} x_{t-1}+\varepsilon_{t} .
$$

That this implies a change in the intercept becomes evident by rewriting the equation as

$$
y_{t}=\beta_{0 t}^{*}+\beta_{1} y_{t-1}+\varepsilon_{t}, \text { with } \beta_{0 t}^{*}=\beta_{0}+\beta_{2} x_{t-1} .
$$

The contemporaneous effect of the covariate can be studied by replacing $x_{t-1}$ by $x_{t}$ in these equations. While the innovation assumptions mentioned for the AR(1) model without covariates remain, the innovations at time $t$ should also be independent of the covariate(s) at time $t-1$ (for lagged effects) or time $t$ (for contemporaneous effects). This - as well as $y_{t-1}$ being independent of $\varepsilon_{t}$ - is termed contemporanous exogeneity of the predictors and guarantees asymptotid ${ }^{3}$ unbiasedness when estimating their effects (Wooldridge, 2012, p.349).

Including an interaction effect between the lagged covariate and the lagged predictor clears the way to additionally study how the AR effect changes in function of the covariate scores:

$$
\begin{aligned}
y_{t} & =\beta_{0}+\beta_{1} y_{t-1}+\beta_{2} x_{t-1}+\beta_{3} x_{t-1} y_{t-1}+\varepsilon_{t} \\
& =\left(\beta_{0}+\beta_{2} x_{t-1}\right)+\left(\beta_{1}+\beta_{3} x_{t-1}\right) y_{t-1}+\varepsilon_{t} \\
& =\beta_{0 t}^{*}+\beta_{1 t}^{*} y_{t-1}+\varepsilon_{t}, \text { with } \beta_{0 t}^{*}=\beta_{0}+\beta_{2} x_{t-1} \text { and } \beta_{1 t}^{*}=\beta_{1}+\beta_{3} x_{t-1} .
\end{aligned}
$$

In this paper, we will mostly focus on the model where only the intercept changes, but come back to the model with a changing AR effect in the discussion.

\subsection{Estimation and collinearity of predictors}

So far, we have considered the AR models of interest at the level of the population. However, the true population-level parameters, which are denoted by greek letters, e.g., $\beta_{1}$, are typically unknown and therefore estimated from data. We discuss the case of OLS estimation in the following. To distinguish

\footnotetext{
${ }^{3}$ i.e. as the sample size tends to infinity
} 
parameter estimators or estimates from population-level parameters we use the hat-notation, e.g., $\hat{\beta_{1}}$.

As indicated in the introduction, we can estimate the parameters of AR(1) models with or without time-varying covariates by means of OLS estimation, based on the assumptions about the innovations that were mentioned higher (see also Wooldridge, 2012). Note that the issue at hand (i.e., potentially impaired estimation precision through predictor collinearity) is not specific to OLS estimators 4 since the origin of the collinearity lies in the data-generating structure, not in how the model is estimated. To recapitulate how OLS estimation works, we rewrite the models in matrix form:

$$
\boldsymbol{y}=\boldsymbol{X} \boldsymbol{\beta}+\boldsymbol{\varepsilon} .
$$

Hereby, $\boldsymbol{y}$ and $\boldsymbol{\varepsilon}$ are $T \times 1$ vectors containing the $T$ scores $y_{t}$ and innovations respectively, $\boldsymbol{X}$ is the $T \times(P+1)$ design matrix, containing a first column of 1's for the intercept and $P$ additional columns for the $P$ predictors and $\beta$ is a $(P+1) \times 1$ vector containing the intercept and the regression weights. For the AR(1) model without covariates, the design matrix thus has two columns, the column of 1's and a column containing the lagged outcome values $y_{t-1}$. When including a covariate to model changes in intercept, we append an additional column to the design matrix, containing the lagged $x_{t-1}$ or contemporaneous $x_{t}$ values. The OLS estimators for the regression coefficients, $\hat{\boldsymbol{\beta}}$, are then obtained as:

$$
\hat{\boldsymbol{\beta}}=\left(\boldsymbol{X}^{\prime} \boldsymbol{X}\right)^{-1} \boldsymbol{X}^{\prime} \boldsymbol{y}
$$

The asymptotic sampling distribution of the OLS estimators is a multivariate normal distribution with mean vector $\boldsymbol{\mu}_{\hat{\boldsymbol{\beta}}}=\boldsymbol{\beta}$ and covariance matrix (Hamilton, 1994, p. 201 f.):

$$
\boldsymbol{\Sigma}_{\hat{\boldsymbol{\beta}}}=\sigma_{\varepsilon}^{2}\left(\boldsymbol{X}^{\prime} \boldsymbol{X}\right)^{-1}
$$

The covariance matrix of this sampling distribution informs us about the variances of each of the

\footnotetext{
${ }^{4}$ For the models considered, OLS and maximum likelihood estimation will lead to the same results asymptotically (Lütkepohl, 2005). We verified (but do not report here) that applying maximum likelihood estimation to our simulated data in section 4 leads to highly similar results.
} 
parameter estimators and their covariances, thus shedding light on estimation precision. The larger the variances, the less precise the obtained estimates are, and the wider the associated confidence intervals will be. In turn, large negative covariances (if they translate to large negative correlations) imply that parameter estimates trade off for each other.

We therefore take a closer look at the OLS estimators' covariance matrix. We first note that it is proportional (by a factor of $\sigma_{\varepsilon}^{2}$ ) to the inverted matrix $\left(\boldsymbol{X}^{\prime} \boldsymbol{X}\right)^{-1}$. The matrix $\boldsymbol{X}^{\prime} \boldsymbol{X}$ in turn is proportional (by a factor of $T$ ) to the covariance matrix of the predictors, $S_{x}$ (including the column of 1 's), plus the products of their means with $\boldsymbol{m}_{\boldsymbol{x}}$ being a $(P+1) \times 1$ vector of predictor means (Ledoit and Wolf, 2003):

$$
\boldsymbol{X}^{\prime} \boldsymbol{X}=T\left(\boldsymbol{S}_{\boldsymbol{x}}+\boldsymbol{m}_{x} \boldsymbol{m}_{\boldsymbol{x}}^{\prime}\right)
$$

From this we can already see that the covariance of the lagged outcome and the lagged or contemporaneous covariate, which is an entry of matrix $S_{x}$ if the covariate is among the predictors, matters for estimation precision. This becomes explicit by deriving the estimator covariance matrix for the AR(1) model with a lagged covariate element-wise, shedding light on the composition of its individual elements, the variances and covariances among the estimators $\hat{\beta_{0}}, \hat{\beta_{1}}, \hat{\beta_{2}}$ in $\hat{\boldsymbol{\beta}}$ (see Appendix A for more detail):

$$
\boldsymbol{\Sigma}_{\hat{\boldsymbol{\beta}}}=\left[\begin{array}{ccc}
\sigma_{\hat{\beta}_{0}}^{2} & \sigma_{\hat{\beta}_{0} \hat{\beta}_{1}} & \sigma_{\hat{\beta_{0}} \hat{\beta}_{2}} \\
\sigma_{\hat{\beta}_{0} \hat{\beta}_{1}} & \sigma_{\hat{\beta_{1}}}^{2}=\frac{\sigma_{\varepsilon}}{T\left(1-r_{y_{t-1} x_{t-1}}^{2}\right) s_{y_{t-1}}^{2}} & \sigma_{\hat{\beta}_{1} \hat{\beta}_{2}}=-\frac{r_{y_{t-1} x_{t-1}} \sigma_{\varepsilon}}{T\left(1-r_{y_{t-1} x_{t-1}}^{2}\right) s_{y_{t-1}} s_{x_{t-1}}} \\
\sigma_{\hat{\beta}_{0} \hat{\beta_{2}}} & \sigma_{\hat{\beta}_{1} \hat{\beta}_{2}}=-\frac{r_{y_{t-1} x_{t-1}} \sigma_{\varepsilon}}{T\left(1-r_{y_{t-1} x_{t-1}}^{2}\right) s_{y_{t-1}} s_{x_{t-1}}} & \sigma_{\hat{\beta_{2}}}^{2}=\frac{\sigma_{\varepsilon}}{T\left(1-r_{y_{t-1} x_{t-1}}^{2}\right) s_{x_{t-1}}^{2}},
\end{array}\right]
$$

Hereby, $s_{x_{t-1}}$ and $s_{y_{t-1}}$ denote the standard deviations of the covariate $x_{t-1}$ and lagged outcome $y_{t-1}$ and $r_{y_{t-1} x_{t-1}}$ denotes their correlation. We can in addition derive that the correlation between the regression weight estimators $\hat{\beta}_{1}$ and $\hat{\beta}_{2}$ equals the predictor correlation in absolute value, but has the opposite sign: 


$$
r_{\hat{\beta}_{1} \hat{\beta}_{2}}=\frac{\sigma_{\hat{\beta}_{1} \hat{\beta}_{2}}}{\sqrt{\sigma_{\hat{\beta_{1}}}^{2}} \sqrt{\sigma_{\hat{\beta}_{2}}^{2}}}=-r_{y_{t-1} x_{t-1}}
$$

Thus, if the correlation between the predictors $r_{y_{t-1} x_{t-1}}$ tends to 1 (i.e., the predictors become highly collinear), the correlation of $\hat{\beta_{1}}$ and $\hat{\beta_{2}}$ will tend to -1 and the variances of these estimators will tend to infinity 5 This points to the crucial role of the predictor correlations in estimation precision. Severe collinearity can thus hamper the interpretation of regression weights (e.g. see Cohen et al., 2013, p. 419). Under high collinearity, slight changes in the data can lead to disproportionate changes in the estimated coefficients (a phenomenon known as bouncing beta behavior (Smith and Sasaki, 1979)). The trade-off behavior between correlated estimates further renders their interpretation as unique direct effects problematic.

Obviously, low predictor correlations are not worrisome, raising the question when predictor correlations are strong enough to be considered indicative of a collinearity problem. Although collinearity naturally is a gradual characteristic, heuristics vary and range from bivariate pedictor correlations exceeding .7 (e.g. Dormann et al., 2013) or .8 (e.g.Slinker and Glantz, 1985, Willis and Perlack, 1978). In this paper we consider any predictor correlation achieving such values as potentially troublesome. Specifically, we will show that the predictor correlations in AR(1) models with time-varying covariates can often reach such levels due to the serial dependence of the variables involved.

\section{COLLINEARITY IN AR(1) MODELS WITH A SINGLE TIME-VARYING COVARI- ATE}

This section presents our analytic results for predictor collinearity in AR(1) models with a single lagged or contemporaneous time-varying covariate. Studying the occurrence of predictor collinearity in this setting, where researchers endow the regression weights with theoretical interpretations, is crucial, because it renders this mapping between theory and statistical parameters shaky. In a first subsection, we present model-implied expressions for the predictor covariance $s_{y_{t-1} x_{t-1}}$ under the model with a lagged and a contemporaneous covariate. In deriving these (see appendix for details), we do not make assumptions regarding how the covariate values were generated. The expressions are thus general

\footnotetext{
${ }^{5}$ In case of perfect collinearity, the model is no longer identifiable since the predictors no longer convey any unique information, and their relative effects can no longer be disentangled (Cohen et al., 2013, p.419)
} 
in the sense that they should accommodate any time-varying covariate. Of course, the presence of predictor covariance alone only tells us that there is collinearity, but not to which degree. Unfortunately, the generality comes at the price that an accessible expression of the predictor correlation cannot straightforwardly be derived. In a second subsection, we therefore turn to the specific case where the time-varying covariate itself follows an AR(1) process. For this case we again show expressions for the model-implied predictor covariance, but also provide one for the predictor correlation in Appendix B.2. In the main text, we visualize the behavior of the predictor covariance and correlation as a function of serial dependence in the covariate, in this case its AR effect, among the other model parameters. We close by briefly discussing the implications of our analytic findings.

\subsection{General expressions}

For the lagged covariate case, we find that the covariance of the predictors $s_{y_{t-1} x_{t-1}}$ can be generally (i.e., for any time-varying covariate) decomposed as (see Appendix B.1):

$$
s_{y_{t-1} x_{t-1}}=\beta_{1} s_{y_{t-2} x_{t-1}}+\beta_{2} s_{x_{t-2} x_{t-1}}+s_{\varepsilon_{t-1} x_{t-1}} .
$$

This expression still features a covariance term concerning both predictors on the right-hand side (i.e., $s_{y_{t-2} x_{t-1}}$. Assuming that our AR(1) process with the lagged covariate effect started a long time ago, and we have obtained sufficiently many observations, we can further decompose this term by recursive substitution. Going $K$ steps back in time, we get:

$$
s_{y_{t-1} x_{t-1}}=\beta_{1}^{K} s_{y_{(t-1)-K} x_{t-1}}+\sum_{k=1}^{K} \beta_{1}^{k-1}\left(\beta_{2} s_{x_{(t-1)-k} x_{t-1}}+s_{\varepsilon_{t-k} x_{t-1}}\right) .
$$

If we take it to the extreme and let $K$ approach $\infty$, then $\beta_{1}^{K}$ approaches zero since $\left|\beta_{1}\right|<1$ (as a necessary condition for stationarity, see section 2.1 ). As a consequence, the term involving the covariance $s_{y_{(t-1)-K} x_{t-1}}$ becomes negligibly small and the expression reduces to:

$$
s_{y_{t-1} x_{t-1}}=\sum_{k=1}^{\infty} \beta_{1}^{k-1}\left(\beta_{2} s_{x_{(t-1)-k} x_{t-1}}+s_{\mathcal{E}_{t-k} x_{t-1}}\right) .
$$

Equation 13 highlights the impact of the autocovariance(s) of the covariate on the predictor covari- 
ance. This obviously also holds for the case where the covariate excerts its influence contemporaneously, in which case we obtain (see Appendix B.1):

$$
s_{y_{t-1} x_{t}}=\beta_{1} s_{y_{t-2} x_{t}}+\beta_{2} s_{x_{t-1} x_{t}}+s_{\varepsilon_{t-1} x_{t}}
$$

and subsequently

$$
s_{y_{t-1} x_{t}}=\sum_{k=1}^{\infty} \beta_{1}^{k-1}\left(\beta_{2} s_{x_{t-k} x_{t}}+s_{\mathcal{E}_{t-k} x_{t}}\right)
$$

\subsection{Expressions when the covariate follows an AR(1) process}

To get more grip on these expressions and the role of the covariate's serial dependence, we studied how the expressions can be rewritten in case the covariate itself is also generated by an $\mathrm{AR}(1)$ process:

$$
x_{t}=\gamma x_{t-1}+\zeta_{t}
$$

with $|\gamma|<1$.

We now distinguish two cases for both lagged and contemporaneous effects: a more general one in which the innovations of $x_{t}, \zeta_{t}$, are contemporaneously related to the innovations of $y_{t}, \varepsilon_{t}$, and a more restricted one in which they are unrelated. In both cases $\zeta_{t}$ and $\varepsilon_{t}$ are jointly normally distributed with a zero mean vector and a covariance matrix that is full in the first case and diagonal in the second case. The variance of $\zeta_{t}$ is denoted by $\sigma_{\zeta}^{2}$, the innovations' covariance by $\left.\sigma_{\zeta \varepsilon}\right]^{6}$

In the more general case of related innovations, the expression for the predictor covariance for the model with the lagged covariate presented in Equation 13 can be rewritten into (see Appendix B.2):

$$
s_{x_{t-1} y_{t-1}}=\frac{\beta_{2} \gamma \frac{\sigma_{\zeta}^{2}}{1-\gamma^{2}}}{1-\left(\beta_{1} \gamma\right)}+\frac{\sigma_{\zeta \varepsilon}}{1-\left(\beta_{1} \gamma\right)} .
$$

Next to $\beta_{1}$ and $\beta_{2}$, the first term of this expression clearly depends on the AR effect of the covariate, $\gamma$, and hence its autocorrelation. The second term contains the covariance of the innovations. In the more restricted case where the innovations are assumed to be uncorrelated (i.e., $\sigma_{\zeta \varepsilon}=0$ ) this

\footnotetext{
${ }^{6} \mathrm{We}$ are thus assuming a specific restricted VAR(1) model in which the lagged effect of the outcome on the covariate is restricted to 0 .
} 
second term obviously drops out. Note that this expression also holds for the model-implied predictor covariance under the model with the contemporaneous covariate (i.e., Equation 15 ; for more detail see Appendix B.2). The expression of the model-implied predictor correlation under both covariate cases can be found in Appendix B.2.

We can use the expression of the predictor covariance (17) and the expression of the predictor correlation $\mathrm{B} .48$ to better understand how both depend on the model parameters $\beta_{1}, \beta_{2}$ and $\gamma$. We visualize this dependence for the predictor covariance in panel A of Figure 1, assuming uncorrelated innovations, an innovation variance $\sigma_{\varepsilon}^{2}$ of 1 , and a covariate variance $\sigma_{x}^{2}$ of 1 . In addition, we also display the corresponding implied predictor correlations in panel B. Figure 1 clearly shows that as $\gamma$ increases, so do the predictor covariances and correlations, in all cases where $\beta_{2} \neq 0$. In addition, if $\gamma=0$ then the predictor covariance remains 0 regardless of $\beta_{1}$ or $\beta_{2}$. Finally, increasing $\beta_{1}$ mainly changes the shape of the functions linking the predictor covariances and correlations to $\gamma$, from more linear shapes to more curved ones, leading to increased covariances and correlations for larger sizes of $\gamma$. Importantly, multiple combinations of $\beta_{1}, \beta_{2}$ and $\gamma$ lead to large predictor correlations (e.g. $\left.r_{x_{t-1} y_{t-1}}>.7\right)$, pointing towards potential collinearity problems.

\subsection{Implications}

We conclude that serial dependence in a covariate that has a non-zero effect $\left(\beta_{2} \neq 0\right)$ on the outcome, will induce (additional) covariance and correlation between the predictors of the AR(1) model with a time-varying covariate. It does thereby not matter whether the covariate originates from a continuous or dichotomous process or is just a deterministic time trend, and whether it is included as contemporaneous or lagged. The implications of this (additional) covariance and correlation are a matter of gradation depending on the sizes of the involved parameters. Our analysis for AR(1) covariates indicates that severe correlations (e.g. $r_{x_{t-1} y_{t-1}}>$.7) will only occur in cases where the true effects underlying the data $\left(\beta_{1}, \beta_{2}\right.$, and particularly $\left.\gamma\right)$ are relatively large (see figure 1 ). In practice, the issue will therefore not always be problematic, although it will still impact estimation precision. To shed further light on when which correlations can be encountered, we will simulate and study different types of covariates in the next section. 
A: model-implied covariances

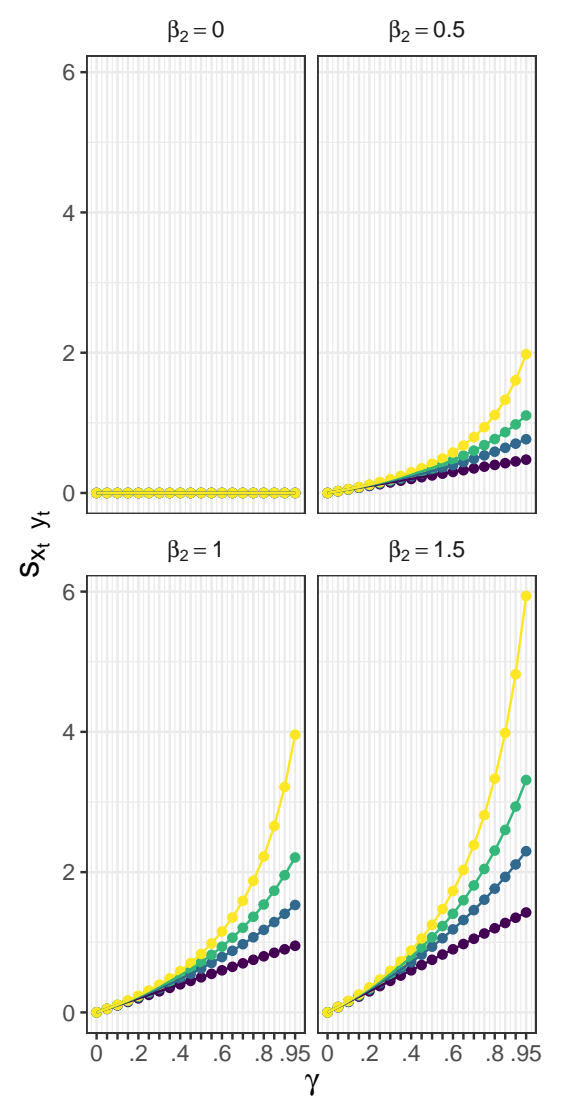

B: model-implied correlations

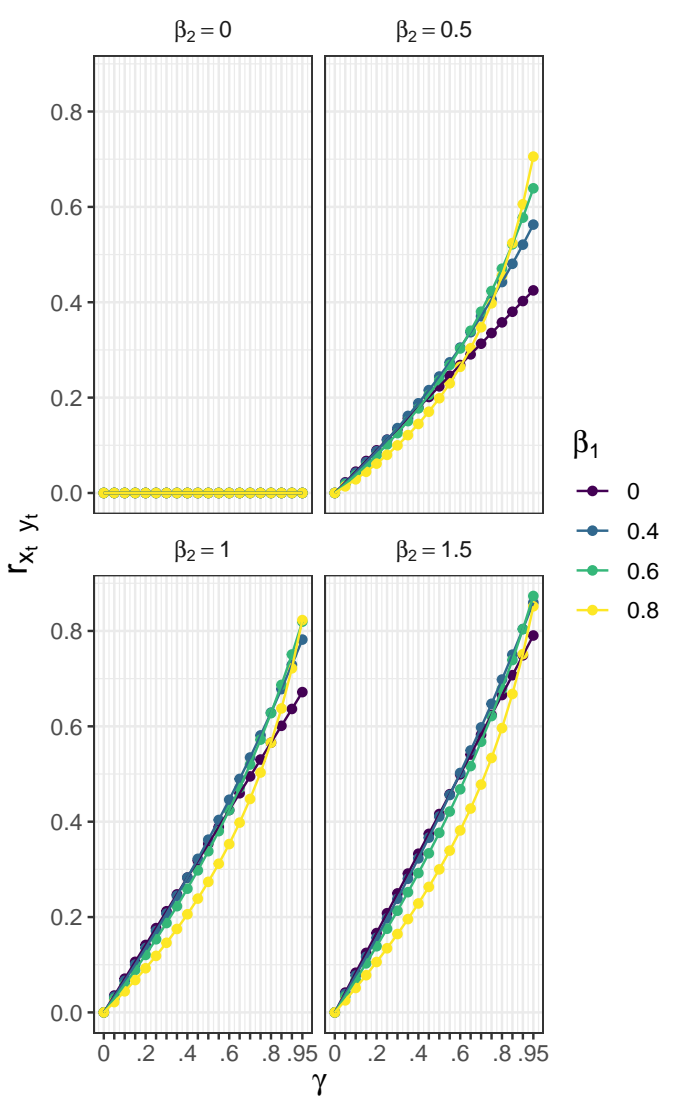

Figure 1. Model-implied predictor covariances (panel A) and predictor correlations (panel B) in case the lagged covariate is generated by an $\mathrm{AR}(1)$ process. The covariances and correlations are depicted as a function of the autocorrelation in the covariate $(\gamma)$, various levels of $\beta_{1}$ (colors) and levels of $\beta_{2}$ (subpanels). 


\section{SIMULATION STUDY}

\subsection{Goals}

We conducted this simulation study with two goals in mind. Firstly we intended to verify our analytic results and showcase that predictor collinearity in AR(1) models with a lagged covariate generalizes beyond continuous covariates (with an $\mathrm{AR}(1)$ structure). We also wanted to illustrate how predictor collinearity depends on the amount of serial dependence in the covariate as well as on the size of $\beta_{1}$ and $\beta_{2}$. To this end, we simulated three different types of covariates and manipulated their serial dependence, as well as $\beta_{1}$ and $\beta_{2}$.

Secondly we aimed to illustrate the impact of predictor collinearity on estimation precision and the extent to which this impact can be alleviated by increasing the number of time points. We predicted that estimation precision would decrease with higher predictor collinearity, but would benefit from larger numbers of time points. All data generation and analysis was performed using $\mathrm{R}$ version 4.0.4 (R Core Team, 2021).

Finally, although we were mainly interested in estimation precision, we also assessed possible biases

in the estimators $\hat{\beta_{1}}$ and $\hat{\beta_{2}}$. As mentioned above, OLS results in asymptotically unbiased estimates of the parameters, but in small samples it is well known that the estimates of the autoregressive effect $\hat{\beta_{1}}$ are biased downwards (Maeshiro, 2000). The extent of this bias has been shown to become negligible for longer time series (Krone et al., 2017).

\subsection{Simulation design and analysis}

We simulated data from the AR(1) model with a time-varying lagged covariate:

$$
y_{t}=\beta_{0}+\beta_{1} y_{t-1}+\beta_{2} x_{t-1}+\varepsilon_{t},
$$

fixing $\beta_{0}$ to 1 and independently drawing all $\varepsilon_{t}$ values from a standard normal distribution. We fully crossed four factors:

1. the number of time points, $T$, with six levels: $50,100,150,250,500,1000$;

2. the size of the AR effect of the lagged outcome, $\beta_{1}$, with three levels: $0, .4, .8$;

3. the size of the effect of the lagged covariate, $\beta_{2}$, with three levels: .5, 1, 1.5;

4. the type of covariate $x_{t}$ and the degree of serial dependence included, with 14 levels; see below. 
Within each of the 756 resulting cells of the design, we generated 1000 datasets, by inserting the obtained covariate values as well as $\beta_{1}$ and $\beta_{2}$ in the model equation above. In all cases, the initial value $y_{0}$ was drawn from the distribution of $\varepsilon_{t}$, and a burn-in period consisting of 500 time points was used.

\subsubsection{Generating covariates}

To assess the consequences of serially dependent covariates and to demonstrate that these consequences generalize to a wide range of possible covariates, we simulated three different types of covariates. Firstly, we generated covariates according to an AR(1) process (accommodating the case discussed in section 3.2), such that $x_{t}=\gamma x_{t-1}+\zeta_{t}$ where $\zeta_{t} \stackrel{i i d}{\sim} N\left(0, \sigma_{\zeta}^{2}\right)$ and with uncorrelated innovations $\zeta_{t}$ and $\varepsilon_{t}$. We fixed $\sigma_{\zeta}^{2}=1$ and varied $\gamma=\{0, .2, .4, .6, .8, .9, .95\}$. Afterwards, the obtained $x_{t}$ scores

were standardized. Since increasing $\gamma$ also increases the variability in the covariate, if $\sigma_{\zeta}^{2}$ is kept constant, this standardization allows us to isolate the consequences of increasing serial dependence in the covariate.

Secondly, we generated dichotomous covariates setting $p_{1}^{x}$, the relative frequency of $x_{t}$ taking on a value of 1 , to .5 . To vary the amount of serial dependence in the covariates, we manipulated the conditional probability $p_{1 \mid 1}^{x}$ of observing $x_{t}=1$ given that $x_{t-1}=1$. This conditional probability is a discrete counterpart of the autocorrelation, as discussed in Bodner et al. (2020) and termed autodependence in the following. Noting that $p_{1 \mid 0}^{x}$, which is the probability of switching from $x_{t-1}=0$ to $x_{t}=1$, can be approximated by:

$$
p_{1 \mid 0}^{x} \approx \frac{p_{1}^{x}-p_{1}^{x} p_{1 \mid 1}^{x}}{1-p_{1}^{x}}
$$

we conclude that the more $p_{1 \mid 0}^{x}$ and $p_{1 \mid 1}^{x}$ deviate from $p_{1}^{x}$, the stronger the autodependence in the covariate. Therefore, we varied $p_{1 \mid 1}^{x}=\{.5, .6, .7, .8, .9, .95\}$. In this case we did not standardize the covariate, to mimic typical dummy-coding of dichotomous predictors in applied research.

Finally, we simulated covariates that reflect a simple linear trend over time, implying an autocorrelation of one. Specifically, we generated $x_{t}=\frac{t}{T}$, and again standardized the resulting scores.

\subsubsection{Analysis}

To verify our analytic results, we first calculated for each generated dataset the predictor variances $\left(s_{y}^{2}\right.$ and $\left.s_{x}^{2}\right)$ and covariance $\left(s_{x_{t-1} y_{t-1}}\right)$. Next, we computed the average values of these statistics within 
each design cell, and used these to obtain an average predictor correlation. Note that this is the proper approach to obtain average correlations, since (independent) co-variances are additive, and can thus be averaged, while correlations are not. For the AR(1) covariate case, where we could derive expressions as a function of the data-generating model parameters, we can thus directly assess whether the modelimplied predictor covariances and correlations adequately describe their counterparts as observed in the simulated (small) samples.

To inspect the impact of time series length and predictor covariance and correlation on estimation precision, we fitted an $\mathrm{AR}(1)$ model with a lagged covariate effect to each generated dataset using OLS by means of the $\operatorname{lm}()$ function in $\mathrm{R}$ base. We computed the variances and correlation of the resulting estimates $\hat{\beta_{1}}$ and $\hat{\beta_{2}}$ within each design cell. Afterwards, the square roots of the average estimator variances per design cell were taken, to obtain average standard errors. Furthermore, we calculated

absolute bias for the estimators $\hat{\beta_{1}}, \hat{\beta_{2}}$ as the average difference between the true and estimated parameter values over replications per design cell. We also obtained relative biases by dividing absolute biases by the respective true parameter value to provide an indication of the proportional extent of the biases.

\subsection{Results}

\subsubsection{Serial dependence and predictor collinearity}

Our first goal was to verify our analytic findings linking serial dependence in the covariate and predictor collinearity. Figure 2 displays the observed predictor covariances (left) and correlations (right) for the AR(1) covariates (Panels A), dichotomous covariates (Panels B), and the trending covariates (Panels C). The covariances and correlations are marked by colored circles with colors reflecting different sizes of $\beta_{1}$ and circle sizes reflecting the different time series lengths. For the $\operatorname{AR}(1)$ covariates, the simulation results are complemented by the model-implied predictor covariances and correlations, marked by red crosses. The simulation results confirm that if the serial dependence in the covariate $(\gamma$ or $p_{1 \mid 1}^{x}$ ) increases, so do the observed predictor covariances and correlations. This confirms that the impact of serial dependence in the covariate on predictor collinearity generalizes over different types of covariates. However, Panels A1 and A2 also reveal discrepancies between the analytical and the observed predictor covariances and correlations, especially for shorter time series. These discrepancies are simply due to the observed (co)variances underestimating the population-level (co)variances, since 
they are calculated relative to the observed means rather than the population-level means (an illustration of this well-known problem can be found in Bishop, 2006, p. 28).

\subsubsection{Estimation precision and small sample biases}

Our second aim was to investigate the impact of predictor collinearity on estimation precision, and the role of the time series length therein. Figure 3 shows that, as expected, the observed predictor correlations are strongly negatively related to the observed estimator correlations, for all covariate types. In addition, Figure 4 displays the expected increase in the estimator standard errors as a function of the serial dependence in the covariate $\left(\gamma\right.$ or $\left.p_{1 \mid 1}^{x}\right)$. As the serial dependence becomes larger, standard errors tend to increase, and this appears most pronounced for the estimate of the covariate effect, $\hat{\beta_{2}}$. Interestingly, large values of $\beta_{1}$ appear to be associated with smaller standard errors for $\hat{\beta_{1}}$ as $\gamma$ increases, but for smaller values of $\beta_{1}$ we still observe a slight increase. In panel $\mathrm{B}$, we observe similar relationships for dichotomous covariates. Panel $\mathrm{C}$ shows that the pattern of increasing standard errors as a function of serial dependence in the covariate that we saw for the AR covariates (panel A) extends to the case where the covariate is perfectly autocorrelated, as in our trending covariate conditions.

Regarding the role of the time series length, figure 4 displays the observed estimator standard errors for time series of 50 and 1000 time points by means of different linetypes (Panels A and B) and along the $\mathrm{x}$-axis (Panel C) respectively. It is evident that the combination of small samples and strongly serially dependent covariates constitute the most problematic cases. For example, for the trending covariate and $T=50, \beta_{1}=0.4$, and $\beta_{2}=1$, the empirical standard error of $\hat{\beta}_{2}$ is larger than that for non-autocorrelated covariates (i.e. AR covariate where $\gamma=0$ ) by a factor of 1.73 .

Finally, figure 5 reveals for small samples, we recover the expected downwards-bias for $\hat{\beta_{1}}$. We also observe a positive bias in $\hat{\beta_{2}}$, which does not appear to be present if $\gamma=0$ (panel A) or $p(1 \mid 1)=0$ (panel $\mathrm{B})$, but increases as $\gamma$ and $p(1 \mid 1)$ increase, suggesting that the bias in $\hat{\beta_{2}}$ originates due to correlation with the biased $\hat{\beta_{1}}$. As expected, the biases disappear in the large $\mathrm{T}$ condition. The magnitude of the biases for $\mathrm{T}=50$ are relatively small, but increase as a function of the autodependence in the covariate. For trending covariates (panel C), the small-sample bias in $\hat{\beta_{2}}$ can be quite pronounced. For example, the relative biases of $\hat{\beta_{2}}$ for $\mathrm{T}=50, \beta_{1}=.4$, over sizes of $\beta_{2}=\{0.5,1,1.5\}$ amount to $.12, .11$, and .08 respectively. For $\beta_{1}=.8$, these relative biases amount to $.34, .20$, and .12 , respectively. 


\section{A: $A R(1)$ covariates}
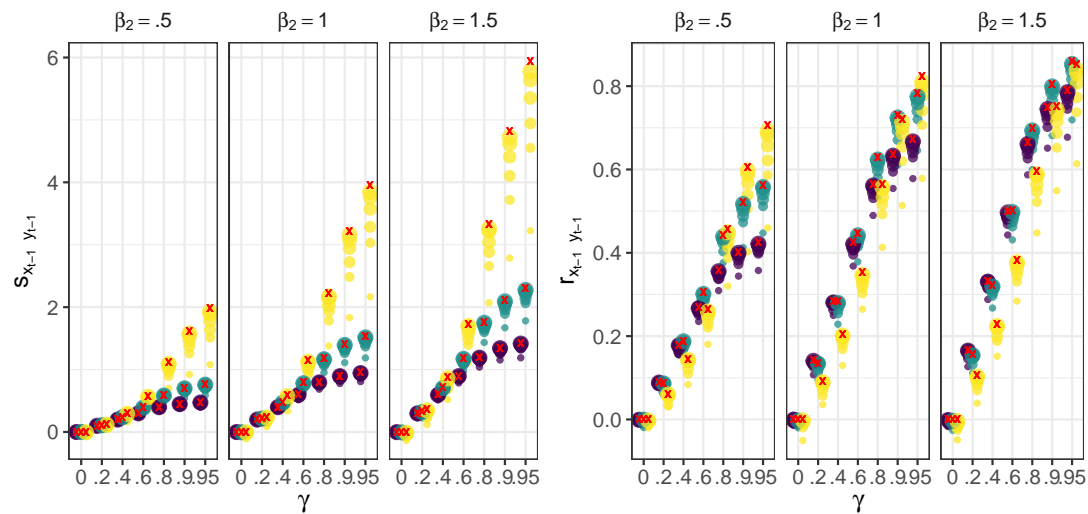

\section{B: Dichotomous covariates}
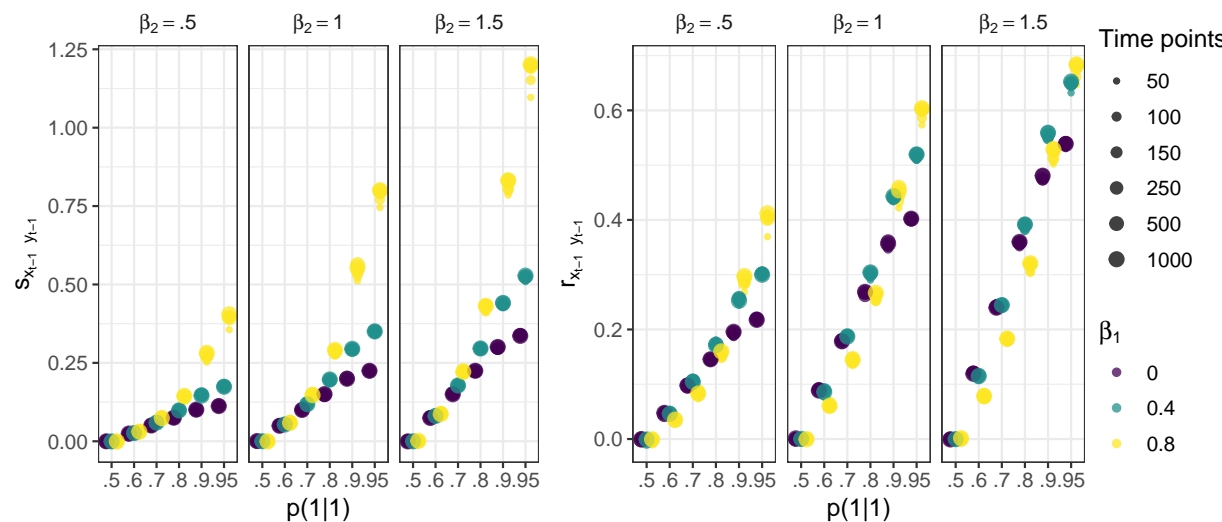

\section{C: Trending covariates}
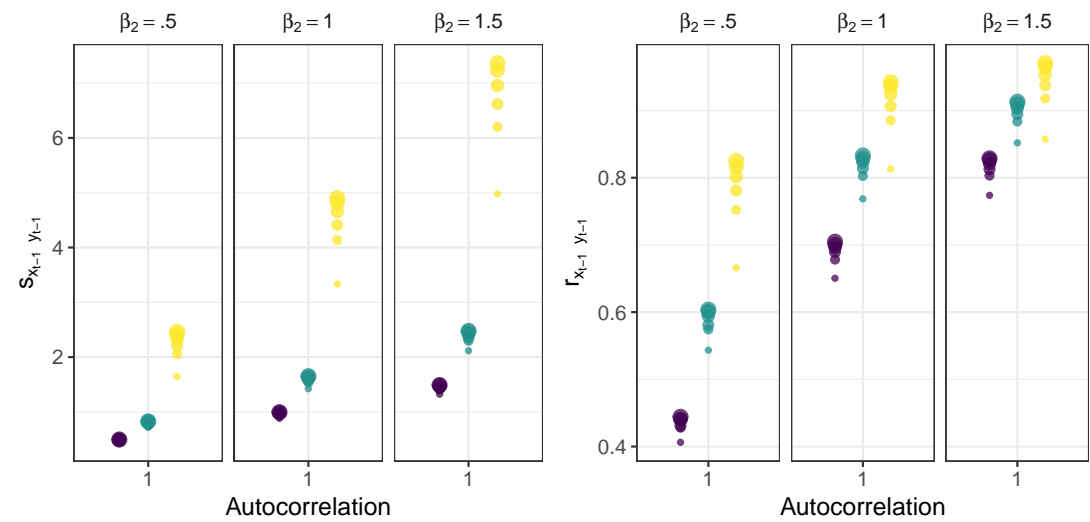

Figure 2. Observed predictor covariances (left) and correlations (right) for the simulated datasets as a function of parameter settings for $\beta_{2}$ (subplots), $\beta_{1}$ (colors), and time points (circle sizes). Panel A displays these statistics for AR(1) covariates, with $\gamma$ on the $\mathrm{x}$-axis. Red crosses correspond to the model-implied relationships for the AR(1) covariate with uncorrelated innovations (expression 17 ). Panel B displays these statistics for dichotomous covariates, with $p(1 \mid 1)$ on the $\mathrm{x}$-axis. Panel $\mathrm{C}$ provides the statistics for the linear trend condition. 

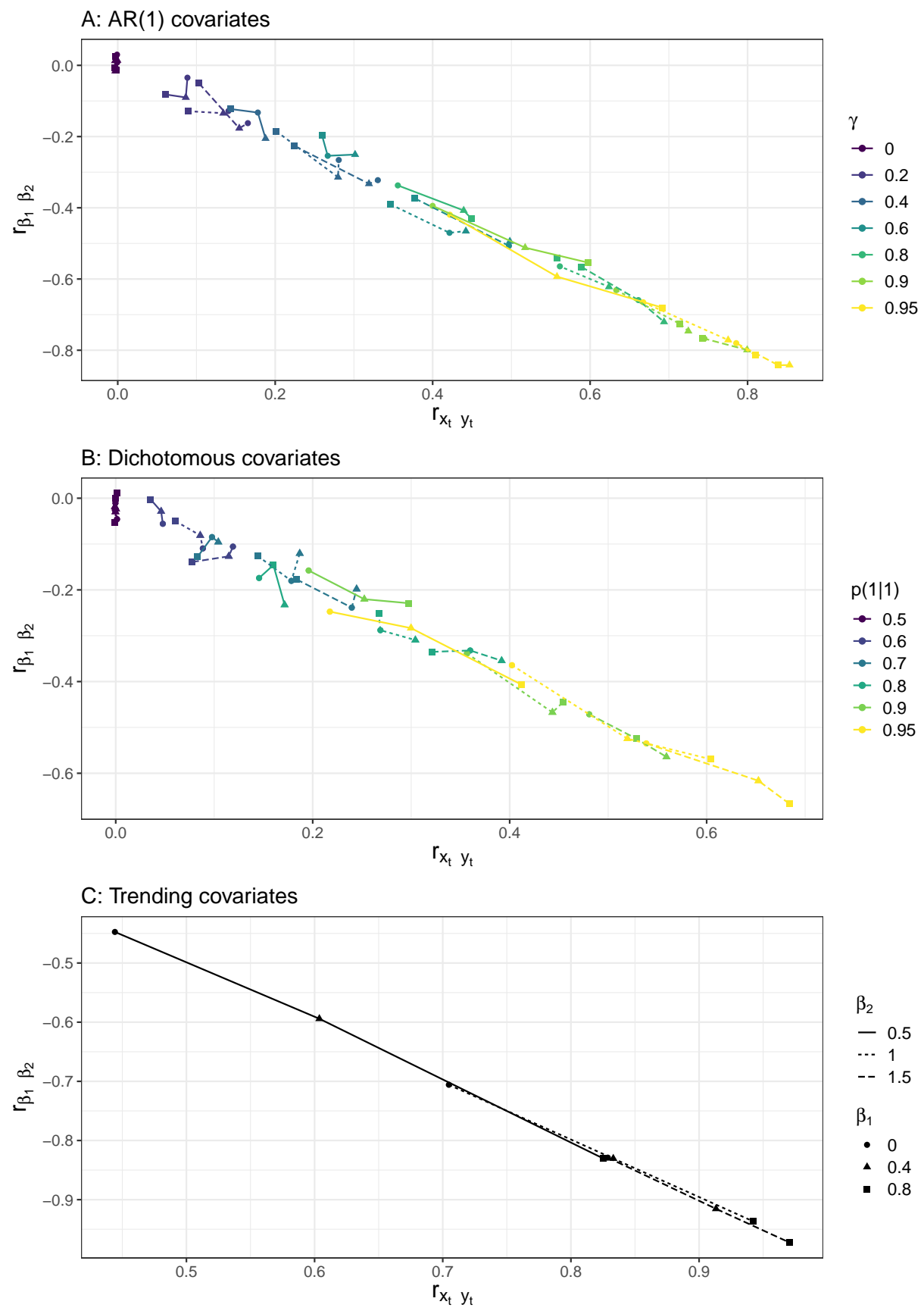

Figure 3. Relationships between the observed predictor correlations (x-axis) and observed estimator correlations (y-axis) as a function of serial dependence in the covariate $(\gamma$ or $p(1 \mid 1))$ for the $\mathrm{AR}(1)$ covariate case (panel A) and dichotomous covariate case (panel B) respectively. Panel C provides the relationship for the trending covariate condition with time series length on the $\mathrm{x}$-axis. Settings for $\beta_{2}$ and $\beta_{1}$ are given by line types and dot-shapes respectively. 
A: $\operatorname{AR}(1)$ covariates

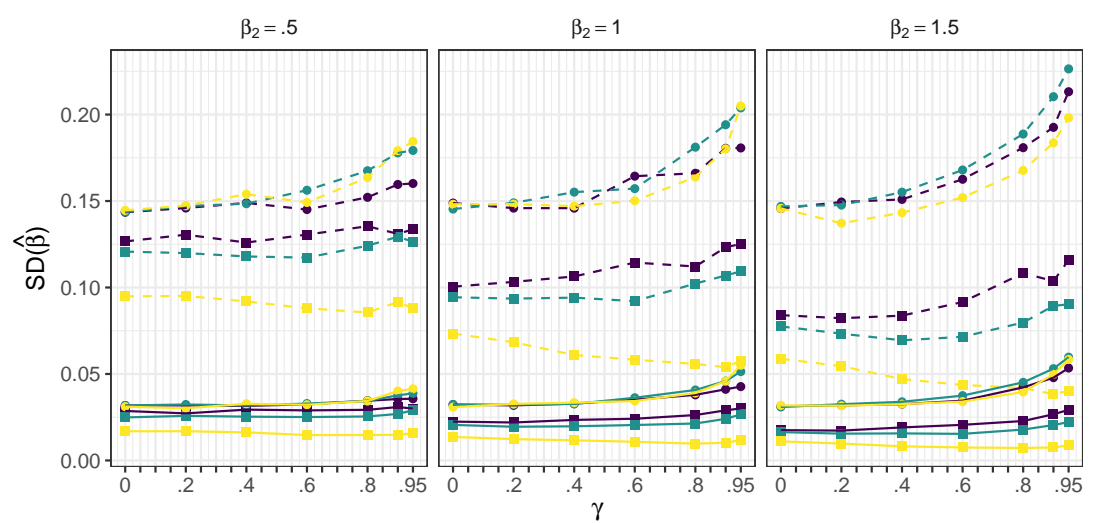

\section{B: Dichotomous covariates}
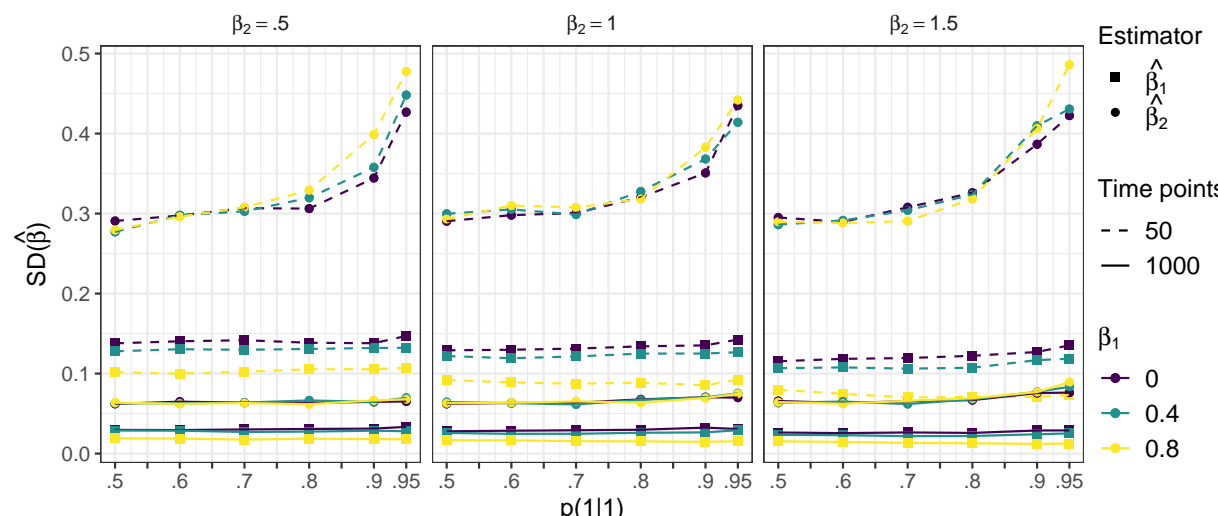

\section{C: Trending covariates}
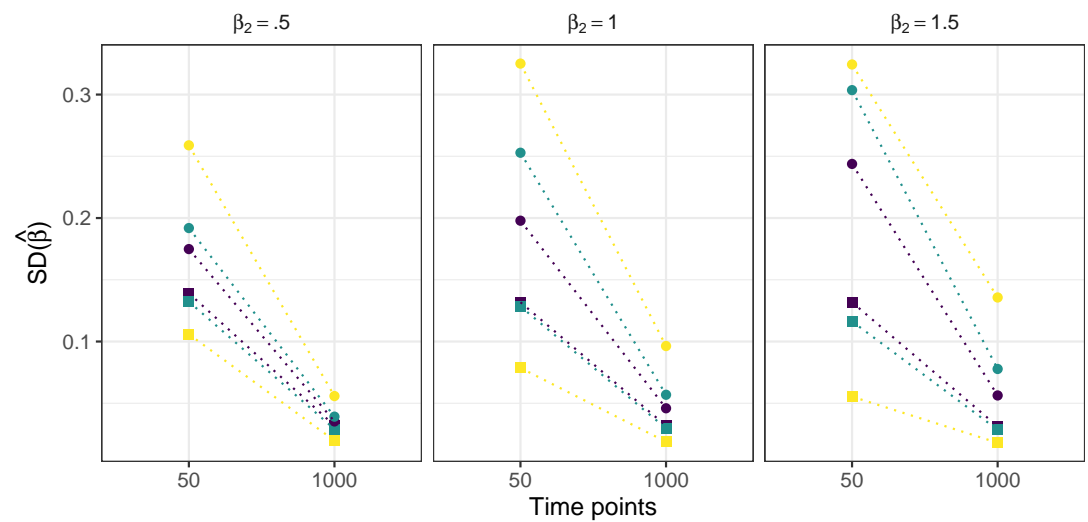

Figure 4. Observed standard deviations (i.e., standard errors) of the estimators (y-axis) $\hat{\beta_{1}}$ (squares) and $\hat{\beta_{2}}$ (dots) as a function of serial dependence in the covariate $(\gamma$ or $p(1 \mid 1)$ ) for the AR(1) covariate case (panel A) and dichotomous covariate case (panel B) respectively. Settings for $\beta_{1}$ are indicated by colors, and the number of simulated time points is indicated by the line type, with solid lines corresponding to the small $(\mathrm{T}=50)$ and large $(\mathrm{T}=1000)$ data sets. Subplots correspond to settings for $\beta_{2}$. Panel $\mathrm{C}$ shows the observed estimator standard errors for small and large simulated data sets (x-axis) for the trend condition. 


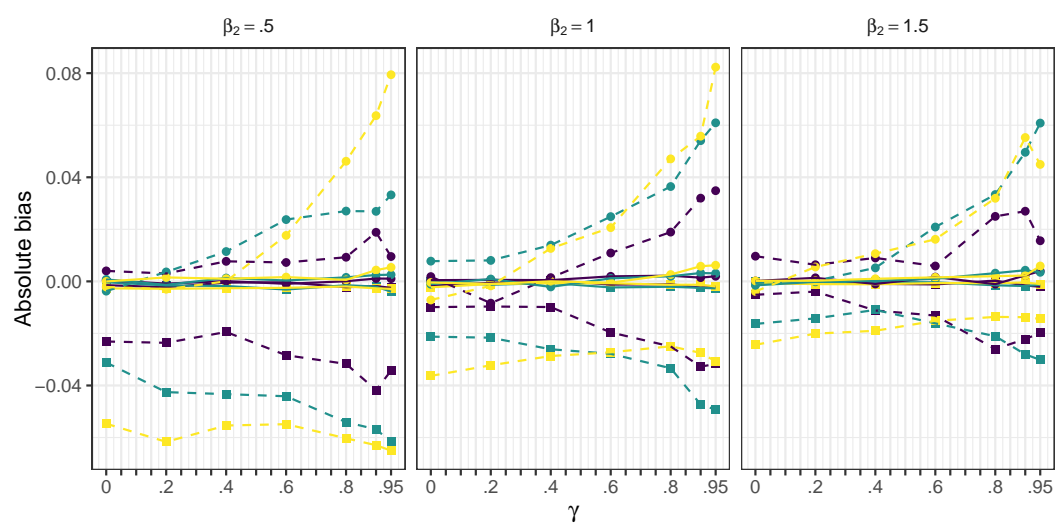

B: Dichotomous covariates
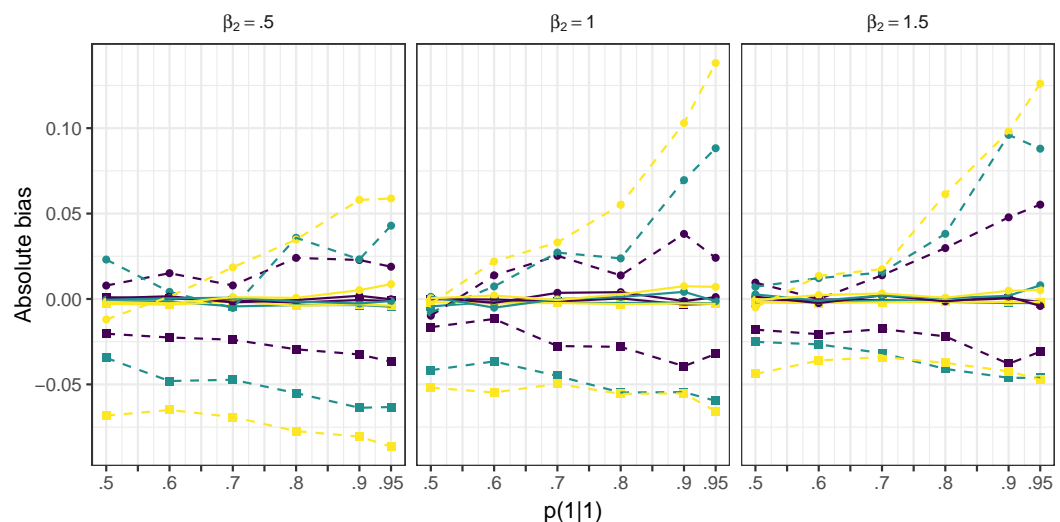

Estimator

- $\hat{\beta_{1}}$

- $\beta_{2}$

Time points

- - 50

$-1000$

$\beta_{1}$

$\rightarrow 0$

$\rightarrow 0.4$

$-0.8$

\section{C: Trending covariates}
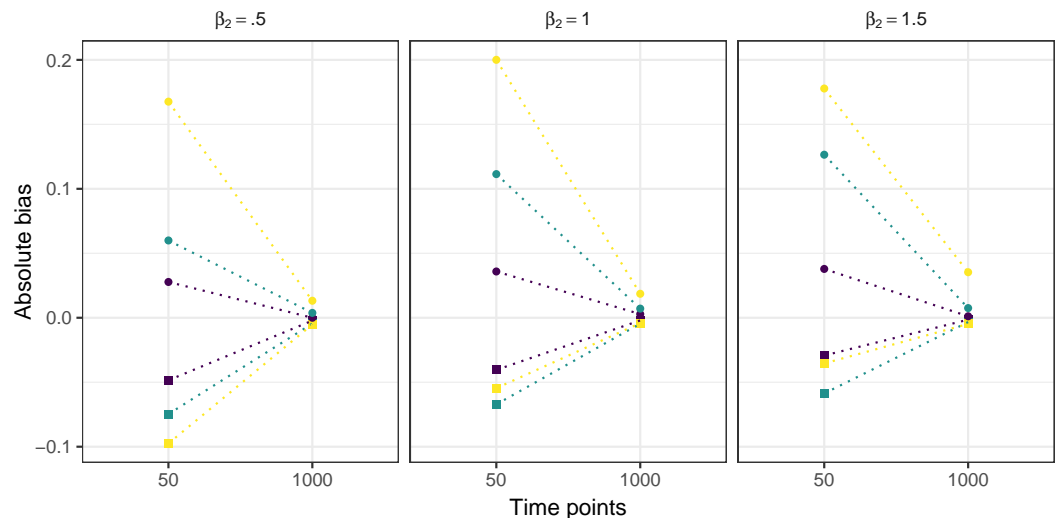

Figure 5. Observed absolute bias of the estimators $\hat{\beta_{1}}$ (squares) and $\hat{\beta_{1}}$ (dots) displayed on the y-axis as a function of the serial dependence in the covariate (i.e., $\gamma$ or $\mathrm{p}(1-1)$ ). Panel A displays the results for the AR covariates, and Panel B for the dichotomous covariates. Panel $\mathrm{C}$ shows the observed absolute bias for small and large conditions (x-axis) for the trend condition. Settings for $\beta_{1}$ are indicated by colors, and the number of simulated time points is indicated by the line type (in Panels A and B), with solid lines corresponding to large $(T=1000)$ and dashed lines corresponding to the small $(T=50)$ simulation conditions. Subplots correspond to different settings for $\beta_{2}$. 


\section{DISCUSSION}

While the sources and consequences of predictor collinearity have received ample attention for crosssectional methods (e.g. Mason and Perreault Jr, 1991, Dormann et al., 2013), we studied the issue in the context of time series analysis, where it has been investigated far less (but see Bulteel et al., 2016, Bulteel et al., 2018). Specifically, this paper examined whether predictor collinearity in AR(1) models with a time-varying covariate can emerge as a function of the serial dependence within the variables involved. Our most important finding is that serial dependence in the covariate, an attribute which is usually not taken into account in applied research, induces (additional) covariance and correlation between the predictors (and hence the lagged outcome and covariate). The severity and thus consequences of such collinearity for applied research - lower estimation precision in the form of larger standard errors and stronger estimator trade-offs - will vary strongly as a function of the various effect sizes underlying the data, and the number of time points at the researchers' disposal. Severe predictor collinearity will typically emerge if AR(1) models contain a covariate that is strongly autocorrelated or trending, and which has a large effect on the process. The decrease in estimation precision will be more pronounced in situations where the model is estimated on a smaller number of time points. The estimator that appears most prone to increased standard errors is the effect of the covariate itself $\left(\hat{\beta}_{2}\right)$. Our simulation results suggest that the small sample bias (in our case $\mathrm{T}=50$ ) for $\hat{\beta}_{1}$ is associated with

a compensatory bias in $\hat{\beta_{2}}$, the extent of which depends on $r_{\hat{\beta}_{1} \hat{\beta}_{2}}$ (and hence indirectly on the serial dependence in the covariate). The remainder of this paper will be dedicated to a number of implications of these findings for applied researchers, focusing on optimal study design and on extensions of the AR(1) model that we studied.

\subsection{Implications for study design}

We want to start by emphasizing that our results should not be interpreted as a recommendation to omit time-varying covariates purely due to concerns about collinearity. If a covariate indeed affects the process under study, failing to include it will lead to biased estimates (e.g. De Boef and Keele, 2008). Then the question obviously becomes how to best handle the issue uncovered. We think the answer lies in optimizing study design, rather than in changing the analysis strategy.

Firstly, one can think about the temporal properties of the time-varying covariate of interest. In experimental studies, one typically has control over this covariate, and manipulates it to produce 
variation in the process of interest. Our results suggest to present experimental stimuli as randomly and void of serial dependence as possible, if permitted by the research question. In such situations, regardless of the magnitude of the effects of interest such as $\beta_{2}$ or $\beta_{1}$, the collinearity problem discussed here will not emerge. For observational studies (e.g., experience sampling studies), this straightforward recommendation is less applicable, since one typically has far less control over the variables under investigation.

In such cases, one can think of other ways to ensure that the effects can be estimated accurately. Since a larger number of time points leads to increased estimation precision, collecting more observations would help in mitigating the consequences of predictor collinearity. Indeed, we saw in the simulation study that including more time points reduces the standard errors of the estimators, also in case of strong serial dependence, and thus also reduces the practical consequences of estimator trade-offs (i.e., high estimator correlations play out less in terms of unstandardized parameter estimates). Since estimation precision is related to power (Brandmaier et al., 2018), this also means that our results have immediate implications for how to run power analyses for the models under study. For instance, Lafit et al. (2021) recently discussed a simulation-based power analysis approach to optimize sample size when one aims to fit multilevel models including AR(1) terms and time-varying covariates. When specifying the data-generating models that are used in the simulation step one should make sure that potential serial dependence in the covariate is taken into account and included when generating data. Otherwise, power calculations might be inaccurate.

\subsection{Implications for model extensions}

\subsubsection{Moderating the autoregressive effect}

We mentioned in the introduction that, next to modeling intercept changes, many researchers justly desire a way of quantifying changes in the AR effect over time, for instance to capture and understand fluctuations in emotional inertia (e.g. Koval and Kuppens, 2012). To this end, researchers can fit a model that contains an interaction effect $\left(\beta_{3} x_{t-1} y_{t-1}\right)$ in addition to the main effects of predictors $x_{t-1}$ and $y_{t-1}$. While we conjecture that this interaction model will also be prone to the collinearity issues discussed in this paper, since it contains our focus model as a special case, the interaction model deserves further attention. Research on cross-sectional methods(e.g., McClelland et al., 2017) indicates that predictor collinearity issues become more complex in such models (e.g., large predictor correlations can sometimes be beneficial for the estimation of the interaction term; McClelland and Judd, 1993, 
Shieh, 2010) and depend, amongst others, on whether or not these predictors are centered (Cohen et al., 2013, p.246, Smith and Sasaki, 1979). Since centering in AR(1) models is a topic in its own right

(E. L. Hamaker and Grasman, 2015) and since researchers usually aim to report and interpret not only the effect of the covariate on the autoregressive effect, $\beta_{3}$, but also $\beta_{1}$ and $\beta_{2}$, the additional impact of serial dependence on the estimation of all three regression weights clearly deserves further study.

\subsubsection{Time polynomials}

Our simulations clearly demonstrated that including time as a linear trend in AR(1) models, which is a popular practice to control for time trends, may lead to collinearity issues, that considerably lower estimation precision when the number of time points is on the small side. In practice, the issue might even get worse, as many researchers try to further control for the often 'wobbly' nature of mean changes in time series by including additional polynomials of time (e.g. $\left.t, t^{2}, t^{3}, \ldots\right)$ as predictors in the model. It is known that polynomial regression models in general suffer from collinearity issues as the polynomial order increases (Shacham and Brauner, 1997). Combining these findings with our results, we hypothesize that models with additional time polynomials will only be more vulnerable to collinearity issues of the here-discussed nature. It might therefore be wise to follow the advice of Michaelides and Spanos, 2020, who argue that a two-stage procedure, in which the regression on time is done prior to estimating the effects of the other predictors, might be a more appropriate way for controlling for such changes over time. Comparing both approaches, two-step and simultaneous, is a useful direction for further research.

\subsubsection{Distributed lags and VAR models}

Next to investigating AR(1) models that include interaction terms or time polynomials, it would also be fruitful to consider extensions of $\mathrm{AR}(1)$ models that include multiple effects of the process variable(s) on themselves. Here, we think of $\operatorname{AR}(p)$ models, which allow to investigate the effect of the previous $p$ process states, as well as VAR(1) models for multivariate processes and the AR distributed lag model (ADL, see Wooldridge, 2012, Harvey, 1989), which includes both lagged and contemporaneous covariate effects. Indeed, both the lagged and contemporaneous models without innovation covariances discussed in this paper could be seen as special cases of ADL models. We also note that the lagged model with an AR(1) covariate corresponds to a restricted VAR(1) model. 


\subsubsection{Multi-subject models}

Whereas this paper has been concerned with the $N=1$ case, many researchers wish to investigate inter-individual differences in intra-individual dynamics and relate these to person-level variables in one way or another. To this end they often apply multilevel or mixed models, where the level 1 equation can also boil down to an $\mathrm{AR}(1)$ process with time-varying covariates (e.g. Gistelinck and Loeys, 2020 , Lafit et al., 2021, Koval and Kuppens, 2012). Studying the potential repercussions of serial dependence in the covariate for such models, seems highly useful. Indeed, if it would turn out that estimating the included random AR and covariate effects would be troublesome in case of high serial dependence in the covariate, this would clearly be important.

\subsection{Conclusion}

While AR(1) models that include time-varying covariates are often used in empirical research, researchers may be unaware of the predictor collinearity that can emerge due to serial dependence in the covariate in combination with moderate to strong AR and covariate effects. The practical consequences of the collinearity are strongly dependent on the number of time points used to estimate the models, however. Therefore, it is always prudent to invest in sufficient time points, and sample size planning should take the (expected) serial dependence in the covariate of interest into account.

\section{REFERENCES}

Adolf, J. K., Voelkle, M. C., Brose, A., \& Schmiedek, F. (2017). Capturing context-related change in emotional dynamics via fixed moderated time series analysis. Multivariate behavioral research, 52(4), 499-531.

Ariens, S., Ceulemans, E., \& Adolf, J. K. (2020). Time series analysis of intensive longitudinal data in psychosomatic research: A methodological overview. Journal of Psychosomatic Research, $137,110191$.

Asparouhov, T., Hamaker, E. L., \& Muthén, B. (2018). Dynamic structural equation models. Structural Equation Modeling: A Multidisciplinary Journal, 25(3), 359-388.

Basinska, B. A., \& Gruszczynska, E. (2020). Burnout as a state: Random-intercept cross-lagged relationship between exhaustion and disengagement in a 10-day study. Psychology research and behavior management, 13, 267-278. 
Beltz, A. M., \& Gates, K. M. (2017). Network mapping with gimme. Multivariate behavioral research, 52(6), 789-804.

Betz, L. T., Penzel, N., Kambeitz-Ilankovic, L., Rosen, M., Chisholm, K., Stainton, A., Haidl, T. K., Wenzel, J., Bertolino, A., Borgwardt, S., et al. (2020). General psychopathology links burden of recent life events and psychotic symptoms in a network approach. npj Schizophrenia, 6(1), $1-8$.

Bishop, C. M. (2006). Pattern recognition and machine learning. Springer.

Bodner, N., Tuerlinckx, F., Bosmans, G., \& Ceulemans, E. (2020). Accounting for auto-dependency in binary dyadic time series data: A comparison of model-and permutation-based approaches for testing pairwise associations. British Journal of Mathematical and Statistical Psychology, 74.

Bosley, H. G., Soyster, P. D., \& Fisher, A. J. (2019). Affect dynamics as predictors of symptom severity and treatment response in mood and anxiety disorders: Evidence for specificity. Journal for Person-Oriented Research, 5(2), 101-113.

Brandmaier, A. M., von Oertzen, T., Ghisletta, P., Lindenberger, U., \& Hertzog, C. (2018). Precision, Reliability, and Effect Size of Slope Variance in Latent Growth Curve Models: Implications for Statistical Power Analysis. Frontiers in Psychology, 9. https://doi.org/10.3389/fpsyg.2018. 00294

Brans, K., Koval, P., Verduyn, P., Lim, Y. L., \& Kuppens, P. (2013). The regulation of negative and positive affect in daily life. Emotion, 13(5), 926-939.

Bringmann, L. F., Ferrer, E., Hamaker, E. L., Borsboom, D., \& Tuerlinckx, F. (2018). Modeling nonstationary emotion dynamics in dyads using a time-varying vector-autoregressive model. Multivariate behavioral research, 53(3), 293-314.

Bringmann, L. F., Hamaker, E. L., Vigo, D. E., Aubert, A., Borsboom, D., \& Tuerlinckx, F. (2017). Changing dynamics: Time-varying autoregressive models using generalized additive modeling. Psychological methods, 22(3), 409-425.

Bulteel, K., Tuerlinckx, F., Brose, A., \& Ceulemans, E. (2016). Using raw var regression coefficients to build networks can be misleading. Multivariate behavioral research, 51(2-3), 330-344.

Bulteel, K., Tuerlinckx, F., Brose, A., \& Ceulemans, E. (2018). Improved insight into and prediction of network dynamics by combining var and dimension reduction. Multivariate behavioral research, 53(6), 853-875. 
Cho, S.-J., Brown-Schmidt, S., \& Lee, W.-y. (2018). Autoregressive generalized linear mixed effect models with crossed random effects: An application to intensive binary time series eye-tracking data. Psychometrika, 83(3), 751-771.

Cohen, J., Cohen, P., West, S. G., \& Aiken, L. S. (2013). Applied multiple regression/correlation analysis for the behavioral sciences (3rd ed.). Routledge.

De Boef, S., \& Keele, L. (2008). Taking time seriously. American journal of political science, 52(1), 184-200.

Degoy, E., \& Olmos, R. (2020). Reciprocal relation between health and academic performance in children through autoregressive models. School Psychology, 35(5), 332-342.

Dormann, C. F., Elith, J., Bacher, S., Buchmann, C., Carl, G., Carré, G., Marquéz, J. R. G., Gruber, B., Lafourcade, B., Leitão, P. J., et al. (2013). Collinearity: A review of methods to deal with it and a simulation study evaluating their performance. Ecography, 36(1), 27-46.

Gistelinck, F., \& Loeys, T. (2020). Multilevel autoregressive models for longitudinal dyadic data. TPM: Testing, Psychometrics, Methodology in Applied Psychology, 27(3), 433-452.

Groen, R. N., Snippe, E., Bringmann, L. F., Simons, C. J., Hartmann, J. A., Bos, E. H., \& Wichers, M. (2019). Capturing the risk of persisting depressive symptoms: A dynamic network investigation of patients' daily symptom experiences. Psychiatry Research, 271, 640-648.

Hamaker, E., Ceulemans, E., Grasman, R., \& Tuerlinckx, F. (2015). Modeling affect dynamics: State of the art and future challenges. Emotion Review, 7(4), 316-322.

Hamaker, E. L., \& Grasman, R. P. (2015). To center or not to center? investigating inertia with a multilevel autoregressive model. Frontiers in psychology, 5, 1492.

Hamilton, J. D. (1994). Time series analysis (Vol. 2). Princeton University Press.

Harvey, A. C. (1989). Explanatory variables. Forecasting, structural time series models and the kalman filter (pp. 365-422). Cambridge University Press.

Houben, M., Van Den Noortgate, W., \& Kuppens, P. (2015). The relation between short-term emotion dynamics and psychological well-being: A meta-analysis. Psychological bulletin, 141(4), 901930.

Jebb, A. T., Tay, L., Wang, W., \& Huang, Q. (2015). Time series analysis for psychological research: Examining and forecasting change. Frontiers in psychology, 6, 727. 
Koval, P., \& Kuppens, P. (2012). Changing emotion dynamics: Individual differences in the effect of anticipatory social stress on emotional inertia. Emotion, 12(2), 256-267.

Koval, P., Pe, M. L., Meers, K., \& Kuppens, P. (2013). Affect dynamics in relation to depressive symptoms: Variable, unstable or inert? Emotion, 13(6), 1132-1141.

Krone, T., Albers, C. J., Kuppens, P., \& Timmerman, M. E. (2018). A multivariate statistical model for emotion dynamics. Emotion, 18(5), 739-754.

Krone, T., Albers, C. J., \& Timmerman, M. E. (2017). A comparative simulation study of ar (1) estimators in short time series. Quality \& quantity, 51(1), 1-21.

Kuppens, P. (2015). It's about time: A special section on affect dynamics. Emotion Review, 7(4), 297-300.

Kuppens, P., Allen, N. B., \& Sheeber, L. B. (2010). Emotional inertia and psychological maladjustment. Psychological science, 21(7), 984-991.

Lafit, G., Adolf, J. K., Dejonckheere, E., Myin-Germeys, I., Viechtbauer, W., \& Ceulemans, E. (2021). Selection of the number of participants in intensive longitudinal studies: A user-friendly shiny app and tutorial for performing power analysis in multilevel regression models that account for temporal dependencies. Advances in Methods and Practices in Psychological Science, 4(1), $1-24$.

Lamers, F., Swendsen, J., Cui, L., Husky, M., Johns, J., Zipunnikov, V., \& Merikangas, K. R. (2018). Mood reactivity and affective dynamics in mood and anxiety disorders. Journal of Abnormal Psychology, 127(7), 659-669.

Leamer, E. E. (1973). Multicollinearity: A bayesian interpretation. The review of economics and statistics, 55, 371-380.

Ledoit, O., \& Wolf, M. (2003). Improved estimation of the covariance matrix of stock returns with an application to portfolio selection. Journal of empirical finance, 10(5), 603-621.

Lütkepohl, H. (2005). New introduction to multiple time series analysis. Springer, Berlin, Heidelberg. Maeshiro, A. (2000). An illustration of the bias of ols for $\mathrm{yt}=\lambda \mathrm{y} \mathrm{t}-1+\mathrm{u}$ t. The Journal of Economic Education, 31(1), 76-80.

Mason, C. H., \& Perreault Jr, W. D. (1991). Collinearity, power, and interpretation of multiple regression analysis. Journal of marketing research, 28(3), 268-280. 
McClelland, G. H., Irwin, J. R., Disatnik, D., \& Sivan, L. (2017). Multicollinearity is a red herring in the search for moderator variables: A guide to interpreting moderated multiple regression models and a critique of iacobucci, schneider, popovich, and bakamitsos (2016). Behavior research methods, 49(1), 394-402.

McClelland, G. H., \& Judd, C. M. (1993). Statistical difficulties of detecting interactions and moderator effects. Psychological bulletin, 114(2), 376-390.

Michaelides, M., \& Spanos, A. (2020). On modeling heterogeneity in linear models using trend polynomials. Economic Modelling, 85, 74-86.

R Core Team. (2021). R: A language and environment for statistical computing. R Foundation for Statistical Computing. Vienna, Austria. https://www.R-project.org/

Schuurman, N. K., Houtveen, J. H., \& Hamaker, E. L. (2015). Incorporating measurement error in n= 1 psychological autoregressive modeling. Frontiers in psychology, 6, 1038.

Shacham, M., \& Brauner, N. (1997). Minimizing the effects of collinearity in polynomial regression. Industrial \& engineering chemistry research, 36(10), 4405-4412.

Shieh, G. (2010). On the misconception of multicollinearity in detection of moderating effects: Multicollinearity is not always detrimental. Multivariate Behavioral Research, 45(3), 483-507.

Simons, J. S., Simons, R. M., Grimm, K. J., Keith, J. A., \& Stoltenberg, S. F. (2020). Affective dynamics among veterans: Associations with distress tolerance and posttraumatic stress symptoms. Emotion, Advance online publication.

Slinker, B., \& Glantz, S. (1985). Multiple regression for physiological data analysis: The problem of multicollinearity. American Journal of Physiology-Regulatory, Integrative and Comparative Physiology, 249(1), R1-R12.

Smith, K. W., \& Sasaki, M. S. (1979). Decreasing multicollinearity: A method for models with multiplicative functions. Sociological Methods \& Research, 8(1), 35-56.

Wang, D., Schneider, S., Schwartz, J. E., \& Stone, A. A. (2020). Heightened stress in employed individuals is linked to altered variability and inertia in emotions. Frontiers in psychology, 11, 1152.

Waugh, C. E., Shing, E. Z., Avery, B. M., Jung, Y., Whitlow, C. T., \& Maldjian, J. A. (2017). Neural predictors of emotional inertia in daily life. Social cognitive and affective neuroscience, 12(9), $1448-1459$. 
Weisstein, E. W. (n.d.). Geometric series. Retrieved July 15, 2021, from https://mathworld.wolfram. com/GeometricSeries.html

Willis, C. E., \& Perlack, R. D. (1978). Multicollinearity: Effects, symptoms, and remedies. Journal of the Northeastern Agricultural Economics Council, 7(1), 55-61.

Wolfram Research, I. (2020). Mathematica (Version 12.1). Champaign, Illinois.

Wooldridge, J. M. (2012). Basic regression analysis with time series data. Introductory econometrics: A modern approach (5th ed., pp. 344-380). Cengage learning.

\section{A COVARIANCE MATRIX OF THE OLS ESTIMATORS FOR THE AR(1) MODEL WITH A TIME-VARYING LAGGED COVARIATE}

In this appendix, we derive the asymptotic covariance matrix of the OLS estimators under the AR(1) model with a time-varying lagged covariate:

$$
y_{t}=\beta_{0}+\beta_{1} y_{t-1}+\beta_{2} x_{t-1}+\varepsilon_{t} .
$$

For this model, the design matrix $\boldsymbol{X}$ contains a column of 1's, a column of lagged-outcome values, and a column of lagged covariate values:

$$
\boldsymbol{X}=\left[\begin{array}{ccc}
1 & y_{0} & x_{0} \\
1 & y_{1} & x_{1} \\
1 & y_{2} & x_{2} \\
\vdots & \vdots & \vdots \\
1 & y_{T-1} & x_{T-1}
\end{array}\right]
$$

Given that OLS estimators cannot handle missing predictor values, there are different strategies of obtaining the initial values $y_{0}$ and $x_{0}$. One might for instance omit the first observation of the outcome variable when fitting the model or impute values for the predictors. For somewhat longer time series, however, it will not have much of an effect which approach one chooses.

Generally, we know that for any $T \times(P+1)$ design matrix (Ledoit and Wolf, 2003): 


$$
\boldsymbol{X}^{\prime} \boldsymbol{X}=T\left(\boldsymbol{S}_{\boldsymbol{x}}+\boldsymbol{m}_{x} \boldsymbol{m}_{\boldsymbol{x}}^{\prime}\right)
$$

This implies that each individual element $\{m, n\}$ of $\boldsymbol{X}^{\prime} \boldsymbol{X}$ equals

$$
\begin{aligned}
\boldsymbol{X}^{\prime} \boldsymbol{X}_{m, n} & =\boldsymbol{x}^{m \prime} \boldsymbol{x}^{n} \\
& =\sum_{t=1}^{T} x_{t}^{m} x_{t}^{n} \\
& =x_{1}^{m} x_{1}^{n}+x_{2}^{m} x_{2}^{n}+\cdots+x_{T}^{m} x_{T}^{n} \\
& =T\left(s_{x^{m}} x^{n}+m_{x^{m}} m_{x^{n}}\right),
\end{aligned}
$$

where $\boldsymbol{x}^{m}$ is the $m$-th column vector of design matrix $\boldsymbol{X}, \boldsymbol{x}^{n}$ is the $n$-th column vector, $s_{x^{m}} x^{n}$ is the covariance of (the values in the) vectors $\boldsymbol{x}^{m}$ and $\boldsymbol{x}^{n}$ and $m_{x^{m}}$ and $m_{x^{n}}$ are their arithmetic means.

For the above-considered model, matrix $\boldsymbol{X}^{\prime} \boldsymbol{X}$ thus looks as follows:

$$
\boldsymbol{X}^{\prime} \boldsymbol{X}=\left[\begin{array}{ccc}
T & T m_{y_{t-1}} & T m_{x_{t-1}} \\
T m_{y_{t-1}} & T\left(s_{y_{t-1}}^{2}+m_{y_{t-1}}^{2}\right) & T\left(s_{y_{t-1} x_{t-1}}+m_{y_{t-1}} m_{x_{t-1}}\right) \\
T m_{x_{t-1}} & T\left(s_{y_{t-1} x_{t-1}}+m_{y_{t-1}} m_{x_{t-1}}\right) & T\left(s_{x_{t-1}}^{2}+m_{x_{t-1}}^{2}\right)
\end{array}\right] .
$$

Writing the covariance of vectors $\boldsymbol{y}_{t-1}$ and $\boldsymbol{x}_{t-1}$ as a function of standard deviations and the correlation, we get:

$$
\boldsymbol{X}^{\prime} \boldsymbol{X}=\left[\begin{array}{ccc}
T & T m_{y_{t-1}} & T m_{x_{t-1}} \\
T m_{y_{t-1}} & T\left(s_{y_{t-1}}^{2}+m_{y_{t-1}}^{2}\right) & T\left(r_{y_{t-1} x_{t-1}} s_{y_{t-1}} s_{x_{t-1}}+m_{y_{t-1}} m_{x_{t-1}}\right) \\
T m_{x_{t-1}} & T\left(r_{y_{t-1} x_{t-1}} s_{y_{t-1}} s_{x_{t-1}}+m_{y_{t-1}} m_{x_{t-1}}\right) & T\left(s_{x_{t-1}}^{2}+m_{x_{t-1}}^{2}\right)
\end{array}\right] .
$$

Via the relationship $\Sigma_{\hat{\boldsymbol{\beta}}}=\sigma_{\varepsilon}^{2}\left(\boldsymbol{X}^{\prime} \boldsymbol{X}\right)^{-1}$ and using Mathematica for symbolic matrix inversion (Wolfram Research, 2020) we get the associated covariance matrix of the OLS estimators $\hat{\boldsymbol{\beta}}=\left(\hat{\beta_{0}}, \hat{\beta_{1}}, \hat{\beta_{2}}\right)^{\prime}$ as: 


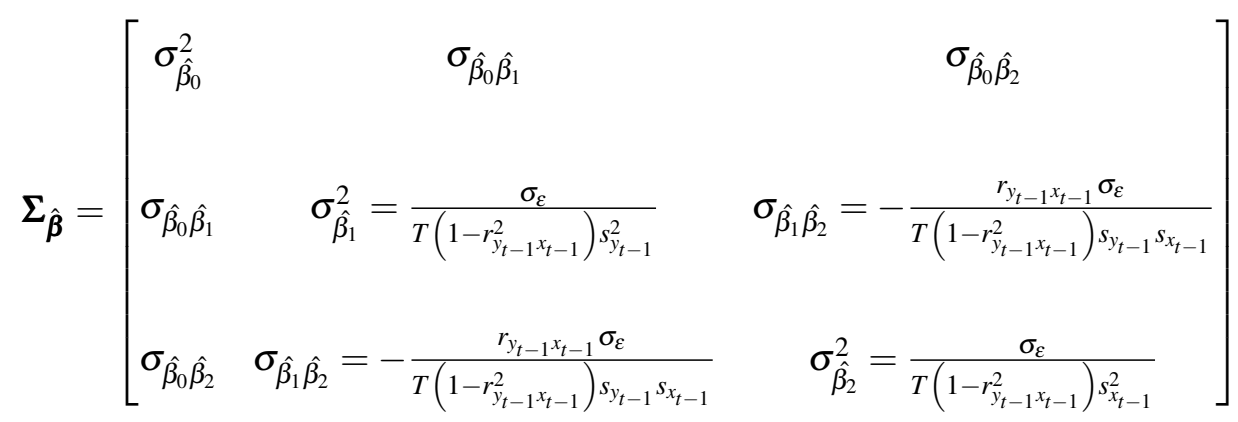

with the following expressions for the entries for the variance and covariances of the intercept estimator:

$$
\begin{aligned}
\sigma_{\hat{\beta}_{0}}^{2} & =-\frac{\sigma_{\varepsilon}\left(-2 r_{y_{t-1} x_{t-1}} m_{y_{t-1}} m_{x_{t-1}} s_{y_{t-1}} s_{x_{t-1}}+s_{x_{t-1}}^{2}\left(m_{y_{t-1}}^{2}-\left(r_{y_{t-1} x_{t-1}}^{2}-1\right) s_{y_{t-1}}^{2}\right)+m_{x_{t-1}}^{2} s_{y_{t-1}}^{2}\right)}{T\left(r_{y_{t-1} x_{t-1}}^{2}-1\right) s_{y_{t-1}}^{2} s_{x_{t-1}}^{2}} \\
\sigma_{\hat{\beta}_{0} \hat{\beta}_{1}} & =\frac{\sigma_{\varepsilon}\left(m_{y_{t-1}} s_{x_{t-1}}-r_{y_{t-1} x_{t-1}} m_{x_{t-1}} s_{y_{t-1}}\right)}{T\left(r_{y_{t-1} x_{t-1}}^{2}-1\right) s_{y_{t-1}}^{2} s_{x_{t-1}}} \\
\sigma_{\hat{\beta}_{0} \hat{\beta}_{2}} & =\frac{\sigma_{\varepsilon}\left(m_{x_{t-1}} s_{y_{t-1}}-r_{y_{t-1} x_{t-1}} m_{y_{t-1}} s_{x_{t-1}}\right)}{T\left(r_{y_{t-1} x_{t-1}}^{2}-1\right) s_{y_{t-1}} s_{x_{t-1}}^{2}} .
\end{aligned}
$$

The correlation between the regression weight estimators can then be obtained as:

$$
\begin{aligned}
\rho_{\hat{\beta}_{1} \hat{\beta}_{2}} & =\frac{\sigma_{\hat{\beta}_{1} \hat{\beta}_{2}}}{\sqrt{\sigma_{\hat{\beta_{1}}}^{2}} \sqrt{\sigma_{\hat{\beta_{2}}}^{2}}} \\
& =-r_{y_{t-1} x_{t-1}} \frac{\sigma_{\varepsilon}}{T\left(1-r_{y_{t-1} x_{t-1}}^{2}\right) s_{y_{t-1}} s_{x_{t-1}} \sqrt{\frac{\sigma_{\varepsilon}}{T\left(1-r_{y_{t-1} x_{t-1}}^{2}\right) s_{y_{t-1}}^{2}}} \sqrt{\frac{\sigma_{\varepsilon}}{T\left(1-r_{y_{t-1}}^{2} x_{t-1}\right) s_{x_{t-1}}^{2}}}} \\
& =-r_{y_{t-1} x_{t-1}} \frac{\sigma_{\varepsilon}}{T\left(1-r_{y_{t-1} x_{t-1}}^{2}\right) s_{y_{t-1}} s_{x_{t-1}} \sqrt{\sigma_{\varepsilon}} \sqrt{\frac{1}{T\left(1-r_{y_{t-1} x_{t-1}}^{2}\right)}} \sqrt{\frac{1}{s_{y_{t-1}}^{2}}} \sqrt{\sigma_{\varepsilon}} \sqrt{\frac{1}{T\left(1-r_{y_{t-1} x_{t-1}}^{2}\right)}} \sqrt{\frac{1}{s_{x_{t-1}}^{2}}}} \\
& =-r_{y_{t-1} x_{t-1}} \frac{\sigma_{\varepsilon}}{T\left(1-r_{y_{t-1} x_{t-1}}^{2}\right) s_{y_{t-1}} s_{x_{t-1}} \frac{\sigma_{\varepsilon}}{s_{y_{t-1}} s_{x_{t-1}} T\left(1-r_{y_{t-1} x_{t-1}}^{2}\right)}} \\
& =-r_{y_{t-1} x_{t-1}} \frac{\sigma_{\varepsilon} s_{y_{t-1}} s_{x_{t-1}} T\left(1-r_{y_{t-1} x_{t-1}}^{2}\right)}{T\left(1-r_{y_{t-1} x_{t-1}}^{2}\right) s_{y_{t-1}} s_{x_{t-1}} \sigma_{\varepsilon}} \\
& =-r_{y_{t-1} x_{t-1}}
\end{aligned}
$$




\section{B DECOMPOSING THE COVARIANCE OF THE PREDICTORS}

\section{B.1 The general case of any time-varying lagged covariate}

In this appendix, we further decompose the covariance between the predictor vectors $\boldsymbol{y}_{t-1}$ and $\boldsymbol{x}_{t-1}$, $s_{y_{t-1} x_{t-1}}$. Using Equation A.22, we can write:

$$
\begin{aligned}
\boldsymbol{y}_{t-1}^{\prime} \boldsymbol{x}_{t-1} & =T\left(s_{y_{t-1} x_{t-1}}+m_{y_{t-1}} m_{x_{t-1}}\right) \\
\Leftrightarrow \quad s_{y_{t-1} x_{t-1}} & =\frac{1}{T} \boldsymbol{y}_{t-1}^{\prime} \boldsymbol{x}_{t-1}-m_{y_{t-1}} m_{x_{t-1}} \\
s_{y_{t-1} x_{t-1}} & =\frac{1}{T} \boldsymbol{y}_{t-1}^{\prime} \boldsymbol{x}_{t-1}-\frac{1}{T}\left(\boldsymbol{y}_{t-1}^{\prime} \mathbf{1}\right) \frac{1}{T}\left(\boldsymbol{x}_{t-1}^{\prime} \mathbf{1}\right),
\end{aligned}
$$

where 1 is a column vector of 1 's. Without loss of generality we assume that $\boldsymbol{x}_{t-1}$ is centered so that the above expression simplifies to:

$$
s_{y_{t-1} x_{t-1}}=\frac{1}{T} \boldsymbol{y}_{t-1}^{\prime} \boldsymbol{x}_{t-1}
$$

We now replace $\boldsymbol{y}_{t-1}^{\prime}$ with $\left(\beta_{0} 1+\beta_{1} \boldsymbol{y}_{t-2}+\beta_{2} \boldsymbol{x}_{t-2}+\boldsymbol{\varepsilon}_{t-1}\right)^{\prime}$ and solve:

$$
\begin{aligned}
s_{y_{t-1} x_{t-1}} & =\frac{1}{T} \boldsymbol{y}_{t-1}^{\prime} \boldsymbol{x}_{t-1} \\
& =\frac{1}{T}\left(\beta_{0} \mathbf{1}+\beta_{1} \boldsymbol{y}_{t-2}+\beta_{2} \boldsymbol{x}_{t-2}+\boldsymbol{\varepsilon}_{t-1}\right)^{\prime} \boldsymbol{x}_{t-1} \\
& =\frac{1}{T}\left(\beta_{0} \mathbf{1}^{\prime}+\beta_{1} \boldsymbol{y}_{t-2}^{\prime}+\beta_{2} \boldsymbol{x}_{t-2}^{\prime}+\boldsymbol{\varepsilon}_{t-1}^{\prime}\right) \boldsymbol{x}_{t-1} \quad \text { | } \\
& =\frac{1}{T}\left(\beta_{0} \mathbf{1}^{\prime} \boldsymbol{x}_{t-1}+\beta_{1} \boldsymbol{y}_{t-2}^{\prime} \boldsymbol{x}_{t-1}+\beta_{2} \boldsymbol{x}_{t-2}^{\prime} \boldsymbol{x}_{t-1}+\boldsymbol{\varepsilon}_{t-1}^{\prime} \boldsymbol{x}_{t-1}\right) \\
& =\frac{1}{T}\left(\beta_{1} \boldsymbol{y}_{t-2}^{\prime} \boldsymbol{x}_{t-1}+\beta_{2} \boldsymbol{x}_{t-2}^{\prime} \boldsymbol{x}_{t-1}+\boldsymbol{\varepsilon}_{t-1}^{\prime} \boldsymbol{x}_{t-1}\right) \quad \mid \mathbf{1}^{\prime} \boldsymbol{x}_{t-1}=0 \text { sinstitute } \\
& =\beta_{1} \frac{1}{T} \boldsymbol{y}_{t-2}^{\prime} \boldsymbol{x}_{t-1}+\beta_{2} \frac{1}{T} \boldsymbol{x}_{t-2}^{\prime} \boldsymbol{x}_{t-1}+\frac{1}{T} \boldsymbol{\varepsilon}_{t-1}^{\prime} \boldsymbol{x}_{t-1} \\
& =\beta_{1} s_{y_{t-2} x_{t-1}+\beta_{2} s_{x_{t-2} x_{t-1}}+s_{\varepsilon_{t-1} x_{t-1}}}
\end{aligned}
$$

This expression still features a covariance term concerning both predictors, $s_{y_{t-2} x_{t-1}}$. To further decompose this term, we again use recursive substitution: In $s_{y_{t-2} x_{t-1}}=\frac{1}{T} \boldsymbol{y}_{t-2}^{\prime} \boldsymbol{x}_{t-1}$, we replace $\boldsymbol{y}_{t-2}^{\prime}$ with $\left(\beta_{0} \mathbf{1}+\beta_{1} \boldsymbol{y}_{t-3}+\beta_{2} \boldsymbol{x}_{t-3}+\boldsymbol{\varepsilon}_{t-2}\right)^{\prime}$ and get:

$$
s_{y_{t-2} x_{t-1}}=\beta_{1} s_{y_{t-3} x_{t-1}}+\beta_{2} s_{x_{t-3} x_{t-1}}+s_{\varepsilon_{t-2} x_{t-1}}
$$


Reinserting this into the last line of Equation B.32 yields:

$$
\begin{aligned}
s_{y_{t-1} x_{t-1}} & =\beta_{1}\left(\beta_{1} s_{y_{t-3} x_{t-1}}+\beta_{2} s_{x_{t-3} x_{t-1}}+s_{\mathcal{E}_{t-2} x_{t-1}}\right)+\beta_{2} s_{x_{t-2} x_{t-1}}+s_{\mathcal{E}_{t-1} x_{t-1}} \\
& =\beta_{1}^{2} s_{y_{t-3} x_{t-1}}+\beta_{1} \beta_{2} s_{x_{t-3} x_{t-1}}+\beta_{2} s_{x_{t-2} x_{t-1}}+\beta_{1} s_{\mathcal{E}_{t-2} x_{t-1}}+s_{\mathcal{E}_{t-1} x_{t-1}} .
\end{aligned}
$$

Assuming that our AR(1) process with the covariate effect started a long time ago, and we were able to take sufficiently many observations, we can go an arbitrary number of $K$ steps back in time:

$$
\begin{aligned}
s_{y_{t-1} x_{t-1}=} & \beta_{1}^{2} s_{y_{t-3} x_{t-1}}+\beta_{1} \beta_{2} s_{x_{t-3} x_{t-1}}+\beta_{2} s_{x_{t-2} x_{t-1}}+\beta_{1} s_{\varepsilon_{t-2} x_{t-1}}+s_{\mathcal{E}_{t-1} x_{t-1}} \\
= & \beta_{1}^{3} s_{y_{t-4} x_{t-1}}+\beta_{1}^{2} \beta_{2} s_{x_{t-4} x_{t-1}}+\beta_{1} \beta_{2} s_{x_{t-3} x_{t-1}}+\beta_{2} s_{x_{t-2} x_{t-1}} \\
& \quad+\beta_{1}^{2} s_{\varepsilon_{t-3} x_{t-1}}+\beta_{1} s_{\mathcal{E}_{t-2} x_{t-1}}+s_{\mathcal{E}_{t-1} x_{t-1}} \\
& \quad \vdots \\
= & \beta_{1}^{K} s_{y_{(t-1)-K} x_{t-1}}+\sum_{k=1}^{K}\left(\beta_{1}^{k-1} \beta_{2} s_{x_{(t-1)-k} x_{t-1}}+\beta_{1}^{k-1} s_{\mathcal{E}_{t-k} x_{t-1}}\right) \\
= & \beta_{1}^{K} s_{y_{(t-1)-K} x_{t-1}}+\sum_{k=1}^{K} \beta_{1}^{k-1}\left(\beta_{2} s_{x_{(t-1)-k} x_{t-1}}+s_{\mathcal{E}_{t-k} x_{t-1}}\right) .
\end{aligned}
$$

If $K$ approaches $\infty, \beta_{1}^{K}$ will approach zero since $\left|\beta_{1}\right|<1$, and the expression simplifies to:

$$
s_{y_{t-1} x_{t-1}}=\sum_{k=1}^{\infty} \beta_{1}^{k-1}\left(\beta_{2} s_{x_{(t-1)-k} x_{t-1}}+s_{\mathcal{E}_{t-k} x_{t-1}}\right) .
$$

Note that the presented steps can also be taken to derive the predictor covariance under the model with the contemporaneous covariate. Only the starting point is then a different one, namely $s_{y_{t-1} x_{t}}=\frac{1}{T} \boldsymbol{y}_{t-1}^{\prime} \boldsymbol{x}_{t}$. For the contemporaneous covariate, we thus get:

$$
\begin{aligned}
s_{y_{t-1} x_{t}} & =\beta_{1} s_{y_{t-2} x_{t}}+\beta_{2} s_{x_{t-1} x_{t}}+s_{\mathcal{E}_{t-1} x_{t}}, \\
& =\beta_{1}^{K} s_{y_{(t-1)-K} x_{t}}+\sum_{k=1}^{K} \beta_{1}^{k-1}\left(\beta_{2} s_{x_{t-k} x_{t}}+s_{\mathcal{E}_{t-k} x_{t}}\right), \\
& =\sum_{k=1}^{\infty} \beta_{1}^{k-1}\left(\beta_{2} s_{x_{t-k} x_{t}}+s_{\mathcal{E}_{t-k} x_{t}}\right) .
\end{aligned}
$$

Go $K$ steps back in time Let $K$ approach $\infty$ 


\section{B.2 The special case of an AR(1) covariate}

We now assume that the values in $\boldsymbol{x}_{t}$ are generated by the zero-mean stationary AR(1) process

$$
x_{t}=\gamma x_{t-1}+\zeta_{t}
$$

with $|\gamma|<1$. For the innovations $\zeta_{t}$ we assume that they are jointly normally distributed with the innovations $\varepsilon_{t}$ which feed into $y_{t}$ (see Equation A.19):

$$
\left[\begin{array}{l}
\zeta_{t} \\
\varepsilon_{t}
\end{array}\right] \stackrel{i i d}{\sim} N\left(\left[\begin{array}{l}
0 \\
0
\end{array}\right],\left[\begin{array}{cc}
\sigma_{\zeta}^{2} & \sigma_{\zeta \varepsilon} \\
\sigma_{\zeta \varepsilon} & \sigma_{\varepsilon}^{2}
\end{array}\right]\right) .
$$

The contemporaneous covariance between the innovations, $\sigma_{\zeta \varepsilon}$, can thus be non-zero. Based on these assumptions, we can further simplify the infinite sum given in Equation B.36, as we will show in the following.

From the time series literature (Hamilton, 1994, p. 53), we know that the $k$ th autocovariance of $x_{t}$ equals:

$$
\sigma_{x_{t} x_{t-k}}=\gamma^{k} \frac{\sigma_{\zeta}^{2}}{1-\gamma^{2}}
$$

This $k$ th autocovariance is independent of $t$, so the expression also holds for $t-1$, for instance. Also, using the expected value operator and the Wold representation of the covariate process (i.e., an AR(1) process can be rewritten as a weighted sum of all its innovations up to time $t$ and its mean, which is 0 in this case), we can derive the model-implied covariance of $x_{t-1}$ with the preceding innovations $\varepsilon_{t-k}$ as (in analogy to Hamilton, 1994, p. 53):

$$
\begin{aligned}
\sigma_{\varepsilon_{t-k} x_{t-1}} & =E\left(\varepsilon_{t-k} x_{t-1}\right) \\
& =E\left(\left(\varepsilon_{t-k}\right)\left(\zeta_{t-1}+\gamma \zeta_{t-2}+\gamma^{2} \zeta_{t-3}+\ldots+\gamma^{k-1} \zeta_{t-k}+\ldots\right)\right) \\
& =\gamma^{k-1} E\left(\varepsilon_{t-k} \zeta_{t-k}\right) \\
& =\gamma^{k-1} \sigma_{\zeta \varepsilon}
\end{aligned}
$$

This means that, under the here-considered AR(1) model for the lagged covariate, the model-implied 
analogue to Equation B.36 can be written as

$$
\begin{aligned}
\sigma_{y_{t-1} x_{t-1}} & =\sum_{k=1}^{\infty} \beta_{1}^{k-1}\left(\beta_{2} \gamma^{k} \frac{\sigma_{\zeta}^{2}}{1-\gamma^{2}}+\gamma^{k-1} \sigma_{\zeta \varepsilon}\right) \\
& =\sum_{k=1}^{\infty}\left(\beta_{1}^{k-1} \beta_{2} \gamma^{k} \frac{\sigma_{\zeta}^{2}}{1-\gamma^{2}}\right)+\sum_{k=1}^{\infty}\left(\beta_{1}^{k-1} \gamma^{k-1} \sigma_{\zeta \varepsilon}\right) \\
& =\frac{\beta_{2}}{\beta_{1}} \frac{\sigma_{\zeta}^{2}}{1-\gamma^{2}} \sum_{k=1}^{\infty}\left(\beta_{1} \gamma\right)^{k}+\frac{\sigma_{\zeta \varepsilon}}{\beta_{1} \gamma} \sum_{k=1}^{\infty}\left(\beta_{1} \gamma\right)^{k} \\
& =\left(\frac{\beta_{2}}{\beta_{1}} \frac{\sigma_{\zeta}^{2}}{1-\gamma^{2}}+\frac{\sigma_{\zeta \varepsilon}}{\beta_{1} \gamma}\right) \sum_{k=1}^{\infty}\left(\beta_{1} \gamma\right)^{k}
\end{aligned}
$$

Since $\left|\beta_{1}\right|<1$ and $|\gamma|<1$, we know that the infinite sum $\sum_{k=1}^{\infty}\left(\beta_{1} \gamma\right)^{k}$ becomes $\frac{\beta_{1} \gamma}{1-\beta_{1} \gamma}$ (Weisstein, n.d.), hence:

$$
\begin{aligned}
\sigma_{y_{t-1} x_{t-1}} & =\left(\frac{\beta_{2}}{\beta_{1}} \frac{\sigma_{\zeta}^{2}}{1-\gamma^{2}}+\frac{\sigma_{\zeta \varepsilon}}{\beta_{1} \gamma}\right) \frac{\beta_{1} \gamma}{1-\beta_{1} \gamma} \\
& =\frac{\beta_{2}}{\beta_{1}} \frac{\sigma_{\zeta}^{2}}{1-\gamma^{2}} \frac{\beta_{1} \gamma}{1-\beta_{1} \gamma}+\frac{\sigma_{\zeta \varepsilon}}{\beta_{1} \gamma} \frac{\beta_{1} \gamma}{1-\beta_{1} \gamma} \\
& =\beta_{2} \gamma \frac{\sigma_{\zeta}^{2}}{1-\gamma^{2}} \frac{1}{1-\beta_{1} \gamma}+\sigma_{\zeta \varepsilon} \frac{1}{1-\beta_{1} \gamma} \\
& =\left(\beta_{2} \gamma \frac{\sigma_{\zeta}^{2}}{1-\gamma^{2}}+\sigma_{\zeta \varepsilon}\right) \frac{1}{1-\beta_{1} \gamma},
\end{aligned}
$$

where $\frac{\sigma_{\zeta}^{2}}{1-\gamma^{2}}$ is the model-implied variance of $x_{t}, \sigma_{x}^{2}$.

Finally, to additionally get an expression of the predictor correlation as a function of the model parameters, we still need to derive the model-implied variance of $y_{t}$ as:

$$
\sigma_{y}^{2}=E\left(y_{t} y_{t}\right)-E\left(y_{t}\right)^{2}
$$


For $E\left(y_{t}\right)^{2}$ we get:

$$
\begin{aligned}
E\left(y_{t}\right)^{2} & =E\left(\beta_{0}+\beta_{1} y_{t-1}+\beta_{2} x_{t-1}+\varepsilon_{t}\right)^{2} \\
& =\left(\beta_{0}+\beta_{1} E\left(y_{t-1}\right)+\beta_{2} E\left(x_{t-1}\right)+E\left(\varepsilon_{t}\right)\right)^{2} \\
& =\left(\beta_{0}+\beta_{1} \mu_{y}\right)^{2} \\
& =\beta_{0}^{2}+2 \beta_{0} \beta_{1} \mu_{y}+\beta_{1}^{2} \mu_{y}^{2},
\end{aligned}
$$

since $x_{t}$ and $\varepsilon_{t}$ are assumed to have zero means. For $E\left(y_{t} y_{t}\right)$ we get:

$$
\begin{aligned}
E\left(y_{t} y_{t}\right)= & E\left(\left(\beta_{0}+\beta_{1} y_{t-1}+\beta_{2} x_{t-1}+\varepsilon_{t}\right)\left(\beta_{0}+\beta_{1} y_{t-1}+\beta_{2} x_{t-1}+\varepsilon_{t}\right)\right) \\
= & E\left(\beta_{0}^{2}+2 \beta_{0} \beta_{1} y_{t-1}+2 \beta_{0} \beta_{2} x_{t-1}+2 \beta_{0} \varepsilon_{t}+\beta_{1}^{2} y_{t-1}^{2}+\beta_{2}^{2} x_{t-1}^{2}\right. \\
& \left.+2 \beta_{1} \beta_{2} y_{t-1} x_{t-1}+2 \beta_{1} y_{t-1} \varepsilon_{t}+2 \beta_{2} x_{t-1} \varepsilon_{t}+\varepsilon_{t}^{2}\right) \\
= & \beta_{0}^{2}+2 \beta_{0} \beta_{1} \mu_{y}+\beta_{1}^{2}\left(\sigma_{y}^{2}+\mu_{y}^{2}\right)+\beta_{2}^{2} \sigma_{x}^{2}+2 \beta_{1} \beta_{2} \sigma_{x y}+\sigma_{\varepsilon}^{2}
\end{aligned}
$$

Plugging the obtained solutions into equation B.44, we obtain:

$$
\begin{aligned}
\sigma_{y}^{2}= & \beta_{0}^{2}+2 \beta_{0} \beta_{1} \mu_{y}+\beta_{1}^{2}\left(\sigma_{y}^{2}+\mu_{y}^{2}\right)+\beta_{2}^{2} \sigma_{x}^{2}+2 \beta_{1} \beta_{2} \sigma_{x y}+\sigma_{\varepsilon}^{2} \\
& -\left(\beta_{0}^{2}+2 \beta_{0} \beta_{1} \mu_{y}+\beta_{1}^{2} \mu_{y}^{2}\right), \\
= & \beta_{1}^{2} \sigma_{y}^{2}+\beta_{2}^{2} \sigma_{x}^{2}+2 \beta_{1} \beta_{2} \sigma_{x y}+\sigma_{\varepsilon}^{2} \\
\Leftrightarrow \quad \sigma_{y}^{2}-\beta_{1}^{2} \sigma_{y}^{2}= & \beta_{2}^{2} \sigma_{x}^{2}+2 \beta_{1} \beta_{2} \sigma_{x y}+\sigma_{\varepsilon}^{2} \\
\left(1-\beta_{1}^{2}\right) \sigma_{y}^{2}= & \beta_{2}^{2} \sigma_{x}^{2}+2 \beta_{1} \beta_{2} \sigma_{x y}+\sigma_{\varepsilon}^{2} \\
\Leftrightarrow \quad \sigma_{y}^{2}= & \frac{\beta_{2}^{2} \sigma_{x}^{2}+2 \beta_{1} \beta_{2} \sigma_{x y}+\sigma_{\varepsilon}^{2}}{1-\beta_{1}^{2}} .
\end{aligned}
$$

Making use of Mathematica (Wolfram Research, 2020), we can now combine this expression and 
the model-implied variance of $x_{t}$ to express the predictor correlation as:

$$
\begin{aligned}
\rho_{x y}= & \frac{\sigma_{x y}}{\sqrt{\sigma_{x}^{2}} \sqrt{\sigma_{y}^{2}}} \\
= & \frac{\sqrt{-\frac{\sigma_{\zeta}^{2}}{\gamma^{2}-1}}\left(\left(\gamma^{2}-1\right) \sigma_{\varepsilon \zeta}-\beta_{2} \gamma \sigma_{\zeta}^{2}\right)}{\left(\beta_{1} \gamma-1\right) \sigma_{\zeta}^{2} \sqrt{\frac{-\beta_{1}\left(-2 \beta_{2}\left(\gamma^{2}-1\right) \sigma_{\varepsilon \zeta}+\beta_{2}^{2} \gamma \sigma_{\zeta}^{2}+\gamma\left(\gamma^{2}-1\right) \sigma_{\varepsilon}^{2}\right)+\beta_{2}^{2}\left(-\sigma_{\zeta}^{2}\right)+\left(\gamma^{2}-1\right) \sigma_{\varepsilon}^{2}}{\left(\beta_{1}^{2}-1\right)\left(\gamma^{2}-1\right)\left(\beta_{1} \gamma-1\right)}}} .
\end{aligned}
$$

Note that the expression for the model-implied predictor covariance presented in Equation B.43 and all following expressions also hold under the model where the covariate exerts a contemporaneous effect, since the $s$ th autocovariance in Equation B.40 is only dependent on $s$ and not on the value $t$ takes. Also, the covariance of the covariate with the preceding innovations derived in Equation B.41 is independent of $t$, as are the model-implied variances of $x_{t}$ and $y_{t}$. 